\title{
UNIVERSALITY AT THE EDGE OF THE SPECTRUM FOR UNITARY, ORTHOGONAL AND SYMPLECTIC ENSEMBLES OF RANDOM MATRICES
}

\author{
PERCY DEIFT AND DIMITRI GIOEV
}

\begin{abstract}
We prove universality at the edge of the spectrum for unitary ( $\beta=$ $2)$, orthogonal $(\beta=1)$ and symplectic $(\beta=4)$ ensembles of random matrices in the scaling limit for a class of weights $w(x)=e^{-V(x)}$ where $V$ is a polynomial, $V(x)=\kappa_{2 m} x^{2 m}+\cdots, \kappa_{2 m}>0$. The precise statement of our results is given in Theorem 1.1 and Corollaries 1.2 1.3 below. For a proof of universality in the bulk of the spectrum, for the same class of weights, for unitary ensembles see [DKMVZ2, and for orthogonal and symplectic ensembles see DG].

Our starting point in the unitary case is [DKMVZ2, and for the orthogonal and symplectic cases we rely on our recent work DG, which in turn depends on the earlier work of Widom [W] and Tracy and Widom [TW2. As in [DG], the uniform Plancherel-Rotach type asymptotics for the orthogonal polynomials found in DKMVZ2 plays a central role.

The formulae in $[\mathrm{W}$ ] express the correlation kernels for $\beta=1$ and 4 as a sum of a Christoffel-Darboux (CD) term, as in the case $\beta=2$, together with a correction term. In the bulk scaling limit $\overline{D G}$, the correction term is of lower order and does not contribute to the limiting form of the correlation kernel. By contrast, in the edge scaling limit considered here, the CD term and the correction term contribute to the same order: this leads to additional technical difficulties over and above DG.
\end{abstract}

\section{INTRODUCTION}

This paper is a continuation of $[\mathrm{DG}$. In $\mathrm{DG}$, the authors proved universality in the bulk for orthogonal and symplectic ensembles: here we prove universality at the edge for orthogonal and symplectic ensembles, and also for unitary ensembles. For the convenience of the reader, and to fix notation, we now summarize some of the basic theory of invariant ensembles $(\beta=1,2$ or 4$)$, borrowing freely and extensively from the introduction in [DG]. We are concerned with ensembles of matrices $\{M\}$ with probability distributions

$$
\mathcal{P}_{N, \beta}(M) d M=\frac{1}{\mathcal{Z}_{N, \beta}} e^{-\operatorname{tr} V_{\beta}(M)} d M,
$$

for $\beta=1,2$ and 4, the so-called Orthogonal, Unitary and Symplectic ensembles, respectively (see M1]). For $\beta=1,2,4$, the ensemble consists of $N \times N$ real symmetric matrices, $N \times N$ Hermitian matrices, and $2 N \times 2 N$ Hermitian selfdual matrices, respectively. In general the potential $V_{\beta}(x)$ is a real-valued function growing sufficiently rapidly as $|x| \rightarrow \infty$, but we will restrict our attention henceforth to $V_{\beta}$ 's which are polynomials,

$$
V_{\beta}(x)=\kappa_{2 m, \beta} x^{2 m}+\cdots, \quad \kappa_{2 m, \beta}>0 .
$$


In (1.1), $d M$ denotes Lebesgue measure on the algebraically independent entries of $M$, and $\mathcal{Z}_{N, \beta}$ is a normalization constant. The above terminology for $\beta=1,2$ and 4 reflects the fact that (1.1) is invariant under conjugation of $M, M \mapsto U M U^{-1}$, by orthogonal, unitary and unitary-symplectic matrices $U$. It follows from (1.1) that the distribution of the eigenvalues $x_{1}, \cdots, x_{N}$ of $M$ is given (see [M1]) by

$$
P_{N, \beta}\left(x_{1}, \cdots, x_{N}\right)=\frac{1}{Z_{N, \beta}} \prod_{1 \leq j<k \leq N}\left|x_{j}-x_{k}\right|^{\beta} \prod_{j=1}^{N} w_{\beta}\left(x_{j}\right)
$$

where again $Z_{N, \beta}$ is a normalization constant (partition function). Here

$$
w_{\beta}(x)= \begin{cases}e^{-V_{\beta}(x)}, & \beta=1,2 \\ e^{-2 V_{\beta}(x)}, & \beta=4 .\end{cases}
$$

(The factor 2 in $w_{\beta=4}$ reflects the fact that the eigenvalues of self-dual Hermitian matrices come in pairs.) Let $\left\{p_{j}\right\}_{j \geq 0}$ be the normalized orthogonal polynomials (OP's) on $\mathbb{R}$ with respect to the weight $w \equiv w_{\beta=2}$, and define $\phi_{j} \equiv p_{j} w^{1 / 2}$. Note that $\left(\phi_{j}, \phi_{k}\right)=\delta_{j k}$ where $(\cdot, \cdot)$ denotes the standard inner product in $L^{2}(\mathbb{R})$.

For the unitary matrix ensembles an important role is played by the ChristoffelDarboux (CD) kernel

$$
K_{N}(x, y) \equiv K_{N, 2}(x, y)=\sum_{k=0}^{N-1} \phi_{k}(x) \phi_{k}(y) .
$$

In particular the probability density (1.3), the $l$-point correlation function $R_{N, l, 2}$ and also the gap probability $E_{2}(0 ; J)$ that a set $J$ contains no eigenvalues, can all be expressed in terms of $K_{N}$, see e.g. [M1]. For example

$$
R_{N, l, 2}\left(x_{1}, \cdots, x_{l}\right)=\operatorname{det}\left(K_{N}\left(x_{j}, x_{k}\right)\right)_{1 \leq j, k \leq l} .
$$

The Universality Conjecture, in our situation, states that the limiting statistical behavior of the eigenvalues $x_{1}, \cdots, x_{N}$ distributed according to the law (1.3), in the appropriate scale as $N \rightarrow \infty$, should be independent of the weight $w_{\beta}$, and should depend only on the invariance properties of $\mathcal{P}_{N, \beta}, \beta=1,2$ or 4 , mentioned above. Universality has been considered extensively in the physics literature, see e.g. BrZ, Be, HWe, SeVe.

The kernel $K_{N}(x, y)$ can also be expressed via the Christoffel-Darboux formula

$$
K_{N}(x, y)=b_{N-1} \frac{\phi_{N}(x) \phi_{N-1}(y)-\phi_{N-1}(x) \phi_{N}(y)}{x-y},
$$

where $b_{N-1}$ is a coefficient in the three-term recurrence relation for OP's, see $\underline{\text { Sz}}$. In view of the preceding remarks it follows that in the case $\beta=2$, the study of the large $N$ behavior of $P_{N, 2}$, and in particular the proof of universality, reduces to the asymptotic analysis of $b_{N-1}$ and the OP's $p_{N+j}$ with $j=0$ or -1 . By a fundamental observation of Fokas, Its and Kitaev [FoIKi] the OP's solve a Riemann-Hilbert problem (RHP) of a type that is amenable to the steepest descent method introduced by Deift and Zhou in [DZ] and further developed in DVZ]. In [DKMVZ1, DKMVZ2] the authors analyzed the asymptotics of OP's for very general classes of weights. In particular they proved the Universality Conjecture in the bulk in the case $\beta=2$ for weights $w(x)=e^{-V(x)}$ where $V(x)$ is a polynomial as above, and also for $w(x)=e^{-N V(x)}$ where $V(x)$ is real analytic and $V(x) / \log |x| \rightarrow+\infty$, as $|x| \rightarrow \infty$. 
The bulk scaling limit as $N \rightarrow \infty$ is described in terms of the so-called sine kernel $K_{\infty}(x-y)$ where

$$
K_{\infty}(t) \equiv \frac{\sin \pi t}{\pi t}
$$

For example DKMVZ2, Theorem 1.4], for $w(x)=e^{-V(x)}, V(x)$ polynomial, and for any $l=2,3, \cdots$ and $r, y_{1}, \cdots, y_{l}$ in a compact set, one has as $N \rightarrow \infty$

$$
\frac{1}{\left(K_{N}(0,0)\right)^{l}} R_{N, l, 2}\left(r+\frac{y_{1}}{K_{N}(0,0)}, \cdots, r+\frac{y_{l}}{K_{N}(0,0)}\right) \rightarrow \operatorname{det}\left(K_{\infty}\left(y_{j}-y_{k}\right)\right)_{1 \leq j, k \leq l} .
$$

The scale $x=y / K_{N}(0,0)$ is chosen so that the expected number of eigenvalues per unit $y$-interval is one. This scaling in the bulk is standard in Random Matrix Theory. Indeed for any Borel set $B \subset \mathbb{R}$,

$$
\int_{B} R_{N, l=1,2}(x) d x=\mathbb{E}\{\text { number of eigenvalues in } B\} .
$$

Thus by (1.6) $K_{N}(0,0)=R_{N, 1,2}(0)$ gives the density of the expected number of eigenvalues near zero. From (1.9), we see that, in the appropriate scale, the large $N$ behavior of the eigenvalues is universal (i.e. independent of $V$ ). Pioneering mathematical work on the Universality Conjecture in the bulk was done in [PS] and for the case of quartic two-interval potential $V(x)=N\left(x^{4}-t x^{2}\right), t>0$ (sufficiently) large, in [BI]. We note again that all these results apply only in the case $\beta=2$.

In the case $\beta=1$ and 4 the situation is more complicated. In place of (1.5) one must use $2 \times 2$ matrix kernels (see e.g. [M1, TW2] )

$$
K_{N, 1}(x, y)=\left(\begin{array}{cc}
S_{N, 1}(x, y) & \left(S_{N, 1} D\right)(x, y) \\
\left(\epsilon S_{N, 1}\right)(x, y)-\frac{1}{2} \operatorname{sgn}(x-y) & S_{N, 1}(y, x)
\end{array}\right), \quad N \text { even, }
$$

and

$$
K_{N, 4}(x, y)=\frac{1}{2}\left(\begin{array}{cc}
S_{N, 4}(x, y) & \left(S_{N, 4} D\right)(x, y) \\
\left(\epsilon S_{N, 4}\right)(x, y) & S_{N, 4}(y, x)
\end{array}\right) .
$$

Here $S_{N, \beta}(x, y), \beta=1,4$, are certain scalar kernels (see (1.17), (1.18) below), $D$ denotes the differentiation operator, and $\epsilon$ is the operator with kernel $\epsilon(x, y)=$ $\frac{1}{2} \operatorname{sgn}(x-y)^{1}$. Such matrix kernels were first introduced by Dyson Dy in the context of circular ensembles with a view to computing correlation functions. Dyson's approach was extended to Hermitian ensembles, first by Mehta $\mathrm{M} 2$ for $V(x)=x^{2}$, and then for more general weights by Mahoux and Mehta in $[\mathrm{MaM}$. A more direct and unifying approach to the results of Dyson-Mahoux-Mehta was given by Tracy and Widom in [TW2, where formulae (1.17), (1.18) below were derived. We see that once the kernels $S_{N, \beta}(x, y)$ are known, then so are the other kernels in $K_{N, \beta}$. As in the case $\beta=2$, the kernels $K_{N, \beta}$ give rise to explicit formulae for $R_{N, l, \beta}$ and $E_{\beta}(0 ; J)$. For example for $\beta=1,4$

$$
R_{N, 1, \beta}(x) \equiv R_{1, \beta}(x)=\frac{1}{2} \operatorname{tr} K_{N, \beta}(x, x)
$$

\footnotetext{
${ }^{1}$ We use the standard notation $\operatorname{sgn} x=1,0,-1$ for $x>0, x=0, x<0$, respectively.
} 
and

$$
R_{N, 2, \beta}(x, y)=\frac{1}{4}\left(\operatorname{tr} K_{N, \beta}(x, x)\right)\left(\operatorname{tr} K_{N, \beta}(y, y)\right)-\frac{1}{2} \operatorname{tr}\left(K_{N, \beta}(x, y) K_{N, \beta}(y, x)\right),
$$

and so on, see [TW2]. We will discuss some of the literature on edge scaling after the statement of our results, Theorem 1.1] Corollary 1.2 and 1.3 below. As indicated above, formula (1.11) only applies to the case when $N$ is even. When $N$ is odd, there is a similar, but slightly more complicated, formula (see [AFNvM]). As in DG], throughout this paper, for $\beta=1$, we will restrict our attention to the case when $N$ is even. We expect that the methods in this paper also extend to the case $\beta=1, N$ odd, and we plan to consider this situation in a later publication. Of course, in situations where the asymptotics of (1.11) has been analyzed (e.g. $V(x)=x^{2}$ ) for all $N$ as $N \rightarrow \infty$, the limiting behavior of $R_{N, l, \beta=1}$ is indeed seen to be independent of the parity of $N$ (see e.g. [M1, [NW]).

Let $\left\{q_{j}(x)\right\}_{j \geq 0}$ be any sequence of polynomials of exact degree $j, q_{j}(x)=q_{j, j} x^{j}+$ $\cdots, q_{j, j} \neq 0$. For $j=0,1,2, \cdots$, set

$$
\psi_{j, \beta}(x)= \begin{cases}q_{j}(x) w_{1}(x), & \beta=1 \\ q_{j}(x)\left(w_{4}(x)\right)^{1 / 2}, & \beta=4 .\end{cases}
$$

Let $M_{N, 1}$ denote the $N \times N$ matrix with entries

$$
\left(M_{N, 1}\right)_{j k}=\left(\psi_{j, 1}, \epsilon \psi_{k, 1}\right), \quad 0 \leq j, k \leq N-1,
$$

and let $M_{N, 4}$ denote the $2 N \times 2 N$ matrix with entries

$$
\left(M_{N, 4}\right)_{j k}=\left(\psi_{j, 4}, D \psi_{k, 4}\right), \quad 0 \leq j, k \leq 2 N-1,
$$

where again $(\cdot, \cdot)$ denotes the standard real inner product on $\mathbb{R}$. The matrices $M_{N, 1}$ and $M_{N, 4}$ are invertible (see e.g. AvM, (4.17), (4.20)]). Let $\mu_{N, 1}, \mu_{N, 4}$ denote the inverses of $M_{N, 1}, M_{N, 4}$ respectively. With these notations we have [TW2 the following formulae for $S_{N, \beta}$ in (1.11), 1.12)

$$
\begin{gathered}
S_{N, 1}(x, y)=-\sum_{j, k=0}^{N-1} \psi_{j, 1}(x)\left(\mu_{N, 1}\right)_{j k}\left(\epsilon \psi_{k, 1}\right)(y) \\
S_{N, 4}(x, y)=\sum_{j, k=0}^{2 N-1} \psi_{j, 4}^{\prime}(x)\left(\mu_{N, 4}\right)_{j k} \psi_{k, 4}(y) .
\end{gathered}
$$

An essential feature of the above formulae is that the polynomials $\left\{q_{j}\right\}$ are arbitrary and we are free to choose them conveniently to facilitate the asymptotic analysis of (1.11), (1.12) as $N \rightarrow \infty$ (see discussion in DG and (1.21) below).

In order to state our main result we need more notation. For any $m \in \mathbb{N}$ let $V(x)$ be a polynomial of degree $2 m$

$$
V(x)=\kappa_{2 m} x^{2 m}+\cdots, \quad \kappa_{2 m}>0
$$

and let $w(x) \equiv w_{\beta=2}(x)=e^{-V(x)}$ as before. Let $p_{j}(x), j \geq 0$, denote the OP's with respect to $w$, and set $\phi_{j}(x) \equiv p_{j}(x)(w(x))^{1 / 2}, j \geq 0$, as above. For $\beta=1,4$ set

$$
V_{\beta}(x) \equiv \frac{1}{2} V(x)
$$


and let $N$ be even. Then by (1.4), $w_{4}=e^{-2 V_{4}}=e^{-V}$ and $w_{1}=e^{-V_{1}}=e^{-V / 2}$. This ensures that for the choice $q_{j}=p_{j}$ in (1.14)

$$
\psi_{j, \beta=1}(x)=\psi_{j, \beta=4}(x)=\phi_{j}(x),
$$

which enables us in turn to handle $S_{N, 1}$ and $S_{N / 2,4}$ in (1.17), (1.18) simultaneously (see [DG, Remark 1.3]). Henceforth and throughout the paper, $K_{N}$ denotes the Christoffel-Darboux (CD) kernel (1.5), (1.7) constructed out of these functions $\phi_{j}$.

For the bulk scaling limit in DKMVZ1 $(\beta=2)$ and DG $(\beta=1,4)$, the authors used the standard scale of one (expected) eigenvalue per unit interval. At the edge it is standard (see e.g. [TW3] to use a slightly different scaling which ensures that the kernel $K_{\text {Airy }}(\xi, \eta)$ (see (1.25) below) appears in the limiting forms (1.26), (1.27), (1.28) below, without any additional factors. Note that formula (1.10) also holds for $\beta=1,4$ and so $R_{N, l=1, \beta}(x)$ gives the density of the expected number of (simple) eigenvalues near $x$ for $\beta=1,2$, 4. In view of (1.10), and also in view of (1.13) and (1.11), (1.12)

$$
R_{N, 1,2}(x)=K_{N}(x, x), \quad R_{N, 1,1}(x)=S_{N, 1}(x, x), \quad R_{N / 2,1,4}(x)=\frac{1}{2} S_{N / 2,4}(x, x) .
$$

To leading order, the right edge of the spectrum is located at the point $c_{N}+d_{N}$ where $c_{N}, d_{N}$ are the Mhaskar-Rakhmanov-Saff numbers in (3.1), (3.2) below. For all three cases, in the neighborhood of $c_{N}+d_{N}$, we use the scale

$$
\xi \mapsto \xi^{(N)} \equiv c_{N}\left(1+\frac{\xi}{\alpha_{N} N^{2 / 3}}\right)+d_{N}
$$

where $\alpha_{N}$ is given in (3.10) (2) below. As we will see (cf. Remark 1.3 below) this scaling differs slightly from a scale of one (expected) eigenvalue per unit interval.

It turns out that the off-diagonal elements in $K_{N, \beta}$ scale differently as $N \rightarrow \infty$. On the other hand, the statistics of the ensembles are clearly invariant (cf. discussion following (2.8) below) under the conjugation

$$
K_{N, \beta} \mapsto K_{N, \beta}^{(\lambda)} \equiv\left(\begin{array}{cc}
\lambda^{-1} & 0 \\
0 & \lambda
\end{array}\right) \cdot K_{N, \beta} \cdot\left(\begin{array}{cc}
\lambda & 0 \\
0 & \lambda^{-1}
\end{array}\right)=\left(\begin{array}{cc}
\left(K_{N, \beta}\right)_{11} & \lambda^{-2}\left(K_{N, \beta}\right)_{12} \\
\lambda^{2}\left(K_{N, \beta}\right)_{21} & \left(K_{N, \beta}\right)_{22}
\end{array}\right)
$$

for any scalar $\lambda$. For example, this is obviously true for the cluster functions $T_{N, l, \beta}$, $\beta=1$ or 4 , which have the form

$$
T_{N, l, \beta}\left(y_{1}, \cdots, y_{l}\right)=\frac{1}{2 l} \sum_{\sigma} \operatorname{tr}\left(K_{N, \beta}\left(y_{\sigma_{1}}, y_{\sigma_{2}}\right) K_{N, \beta}\left(y_{\sigma_{2}}, y_{\sigma_{3}}\right) \cdots K_{N, \beta}\left(y_{\sigma_{l}}, y_{\sigma_{1}}\right)\right)
$$

where the sum is taken over all permutations of $\{1, \cdots, l\}$ (see [TW2 p. 816]), etc.

Denote

$$
\begin{aligned}
K_{\text {Airy }}(\xi, \eta) & \equiv \frac{\operatorname{Ai}(\xi) \operatorname{Ai}^{\prime}(\eta)-\operatorname{Ai}^{\prime}(\xi) \operatorname{Ai}(\eta)}{\xi-\eta} \\
& =\int_{0}^{\infty} \operatorname{Ai}(z+\xi) \operatorname{Ai}(z+\eta) d z
\end{aligned}
$$

Set

$$
\lambda_{(N)} \equiv\left(\frac{c_{N}}{\alpha_{N} N^{2 / 3}}\right)^{-1 / 2}
$$

Theorem 1.1 and Corollary 1.2 and 1.3 below are the main results in this paper. 
Theorem 1.1. Let $\beta=2,1$ or 4 . For any $V(x)$ of degree $2 m$ as in (1.19) define $V_{\beta}(x)$ and $w_{\beta}(x)$ as in (1.20), (1.4). Fix a number $L_{0}$. Then there exists $c=$ $c\left(L_{0}\right)>0$ such that as $N \rightarrow \infty^{2}$ the following holds uniformly for $\xi, \eta \in\left[L_{0},+\infty\right)$.

In the case $\beta=2$ :

$$
\mathcal{E}_{N, 2} \equiv \frac{1}{\lambda_{(N)}^{2}} K_{N}\left(\xi^{(N)}, \eta^{(N)}\right)-K_{\text {Airy }}(\xi, \eta) \rightarrow 0 .
$$

In the case $\beta=1$ :

$$
\mathcal{E}_{N, 1} \equiv \frac{1}{\lambda_{(N)}^{2}} K_{N, 1}^{\left(\lambda_{(N)}\right)}\left(\xi^{(N)}, \eta^{(N)}\right)-K^{(1)}(\xi, \eta) \rightarrow 0
$$

where

$$
\begin{aligned}
\left(K^{(1)}\right)_{11}(\xi, \eta)= & \left(K^{(1)}\right)_{22}(\eta, \xi) \equiv K_{\text {Airy }}(\xi, \eta)+\frac{1}{2} \operatorname{Ai}(\xi) \cdot \int_{-\infty}^{\eta} \operatorname{Ai}(t) d t \\
\left(K^{(1)}\right)_{12}(\xi, \eta) \equiv & -\partial_{\eta} K_{\text {Airy }}(\xi, \eta)-\frac{1}{2} \operatorname{Ai}(\xi) \operatorname{Ai}(\eta) \\
\left(K^{(1)}\right)_{21}(\xi, \eta) \equiv & -\int_{\xi}^{\infty} K_{\text {Airy }}(t, \eta) d t \\
& -\frac{1}{2} \int_{\xi}^{\eta} \operatorname{Ai}(t) d t+\frac{1}{2} \int_{\xi}^{\infty} \operatorname{Ai}(t) d t \cdot \int_{\eta}^{\infty} \operatorname{Ai}(t) d t-\frac{1}{2} \operatorname{sgn}(\xi-\eta) .
\end{aligned}
$$

In the case $\beta=4$ :

$$
\mathcal{E}_{N, 4} \equiv \frac{1}{\lambda_{(N)}^{2}} K_{N / 2,4}^{\left(\lambda_{(N)}\right)}\left(\xi^{(N)}, \eta^{(N)}\right)-K^{(4)}(\xi, \eta) \rightarrow 0
$$

where

$$
\begin{aligned}
& 2\left(K^{(4)}\right)_{11}(\xi, \eta)=2\left(K^{(4)}\right)_{22}(\eta, \xi) \equiv K_{\text {Airy }}(\xi, \eta)-\frac{1}{2} \operatorname{Ai}(\xi) \cdot \int_{\eta}^{\infty} \operatorname{Ai}(t) d t \\
& 2\left(K^{(4)}\right)_{12}(\xi, \eta) \equiv-\partial_{\eta} K_{\text {Airy }}(\xi, \eta)-\frac{1}{2} \operatorname{Ai}(\xi) \operatorname{Ai}(\eta) \\
& 2\left(K^{(4)}\right)_{21}(\xi, \eta) \equiv-\int_{\xi}^{\infty} K_{\text {Airy }}(t, \eta) d t+\frac{1}{2} \int_{\xi}^{\infty} \operatorname{Ai}(t) d t \cdot \int_{\eta}^{\infty} \operatorname{Ai}(t) d t .
\end{aligned}
$$

For the error term we have as $N \rightarrow \infty$

$$
\begin{aligned}
& \mathcal{E}_{N, 2}=O\left(N^{-2 / 3}\right) e^{-c \xi} e^{-c \eta} \\
& \mathcal{E}_{N, 1}=o(1)\left(\begin{array}{cc}
e^{-c \xi} & e^{-c \xi} e^{-c \eta} \\
1 & e^{-c \eta}
\end{array}\right) \\
& \mathcal{E}_{N, 4}=o(1) e^{-c \xi} e^{-c \eta}
\end{aligned}
$$

uniformly for $\xi, \eta \in\left[L_{0},+\infty\right)$.

Remark 1.1. For $\beta=4$, but not for $\beta=1$, our methods actually prove that $\mathcal{E}_{N, 4}=O\left(N^{-1 /(2 m)}\right) e^{-c \xi} e^{-c \eta}$. In order to obtain power law decay for $\mathcal{E}_{N, 1}$, it would be sufficient to obtain power law decay in the error term in [DG, Theorem 2.2]: such power law decay can be obtained using more sophisticated estimates as in [DGKV].

${ }^{2}$ For $\beta=1,4, N$ is even. 
We immediately have the following result. Recall formula (1.24) for the cluster functions for $\beta=1,4$; for $\beta=2$, the cluster functions have the form [TW2, p. 815]

$$
T_{N, l, 2}\left(y_{1}, \cdots, y_{l}\right)=\frac{1}{l} \sum_{\sigma} K_{N}\left(y_{\sigma_{1}}, y_{\sigma_{2}}\right) K_{N}\left(y_{\sigma_{2}}, y_{\sigma_{3}}\right) \cdots K_{N}\left(y_{\sigma_{l}}, y_{\sigma_{1}}\right) .
$$

Corollary 1.2. Let $\beta=2,1$ or 4 . Let $V$ be a polynomial of degree $2 m$ and let $K^{(\beta)}, \beta=1,4$ be as in Theorem 1.1. Fix a number $L_{0}$. Then for $\beta=1$ and $l=2,3, \cdots$ we have uniformly for $\xi_{1}, \cdots, \xi_{l} \geq L_{0}$

$$
\begin{aligned}
\lim _{N \rightarrow \infty} \frac{1}{\left(\lambda_{(N)}^{2}\right)^{l}} T_{N, l, 1}\left(\left(\xi_{1}\right)^{(N)}, \cdots,\left(\xi_{1}\right)^{(N)}\right) \\
\quad=\frac{1}{2 l} \sum_{\sigma} \operatorname{tr}\left(K^{(1)}\left(\xi_{\sigma_{1}}, \xi_{\sigma_{2}}\right) K^{(1)}\left(\xi_{\sigma_{2}}, \xi_{\sigma_{3}}\right) \cdots K^{(1)}\left(\xi_{\sigma_{l}}, \xi_{\sigma_{1}}\right)\right) .
\end{aligned}
$$

For $\beta=4$, the same result is true provided we replace $T_{N, l, 1} \rightarrow T_{N / 2, l, 4}$ and $K^{(1)} \rightarrow$ $K^{(4)}$. For $\beta=2$, the same result is true provided we replace $T_{N, l, 1} \rightarrow T_{N, l, 2}$, $K^{(1)} \rightarrow K_{\text {Airy }}, \frac{1}{2 l} \rightarrow \frac{1}{l}$, and remove the trace.

Together with some additional estimates (see Section 21), Theorem 1.1] also yields the following universality result for the gap probabilities. Recall that for a $2 \times 2$ block operator $A=\left(A_{i j}\right)_{i, j=1,2}$ with $A_{11}, A_{22}$ in trace class and $A_{12}, A_{21}$ Hilbert-

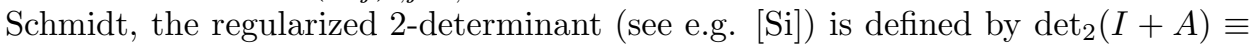
$\operatorname{det}\left((I+A) e^{-A}\right) e^{\operatorname{tr}\left(A_{11}+A_{22}\right)}$.

Let $\lambda_{1}$ denote the largest eigenvalue of a random matrix $M$.

Corollary 1.3. Let $\beta=2,1$ or 4 . Let $V$ be a polynomial of degree $2 m$ and let $K^{(\beta)}, \beta=1,4$ be as in Theorem 1.1. Fix a number $L_{0}$. Then the following holds.

In the case $\beta=2$ :

$$
\lim _{N \rightarrow \infty} \operatorname{Prob}\left\{\lambda_{1} \leq\left(L_{0}\right)^{(N)}\right\}=\operatorname{det}\left(I-\left.K_{\text {Airy }}\right|_{L^{2}\left(\left[L_{0},+\infty\right)\right)}\right) \equiv F^{(2)}\left(L_{0}\right) .
$$

In the case $\beta=4$ :

$$
\lim _{N \rightarrow \infty} \operatorname{Prob}\left\{\lambda_{1} \leq\left(L_{0}\right)^{(N)}\right\}=\sqrt{\left.\left.\operatorname{det}\left(I-K^{(4)}\right)\right|_{L^{2}\left(\left[L_{0},+\infty\right)\right)}\right)} \equiv F^{(4)}\left(L_{0}\right) .
$$

In the case $\beta=1$, let $g(\xi) \equiv \sqrt{1+\xi^{2}}, G=\operatorname{diag}\left(g, g^{-1}\right)$. Then

$$
\lim _{N \rightarrow \infty} \operatorname{Prob}\left\{\lambda_{1} \leq\left(L_{0}\right)^{(N)}\right\}=\sqrt{\operatorname{det}_{2}\left(I-\left.G K^{(1)} G^{-1}\right|_{L^{2}\left(\left[L_{0},+\infty\right)\right)}\right)} \equiv F^{(1)}\left(L_{0}\right) .
$$

Remark 1.2. The regularized 2-determinant is needed for $\beta=1$ because the operator with kernel $\frac{1}{2} \operatorname{sgn}(\xi-\eta)$ is Hilbert-Schmidt but not trace class in $L^{2}\left(\left[L_{0},+\infty\right)\right)$. The auxiliary function $g$ is needed to ensure that $G K^{(1)} G^{-1}$ indeed has a 2determinant: there is considerable freedom in the choice of the function $g$, see Remark 2.2 below.

Remark 1.3. From Theorem 1.1 and (1.22) we have as $N \rightarrow \infty$,

$$
\begin{aligned}
\frac{c_{N}}{\alpha_{N} N^{2 / 3}} R_{N, 1,2}\left(t^{(N)}\right) & =K_{\text {Airy }}(t, t)+o(1) \\
\frac{c_{N}}{\alpha_{N} N^{2 / 3}} R_{N, 1,1}\left(t^{(N)}\right) & =K_{\text {Airy }}(t, t)+\frac{1}{2} \operatorname{Ai}(t) \int_{-\infty}^{t} \operatorname{Ai}(u) d u+o(1) \\
\frac{c_{N}}{\alpha_{N} N^{2 / 3}} R_{N / 2,1,4}\left(t^{(N)}\right) & =\frac{1}{4} K_{\text {Airy }}(t, t)-\frac{1}{8} \operatorname{Ai}(t) \int_{t}^{\infty} \operatorname{Ai}(u) d u+o(1)
\end{aligned}
$$


uniformly for $t$ in any fixed half-line $\left[L_{0},+\infty\right)$. In particular the density of the expected number of eigenvalues at the edge of the spectrum $c_{N}+d_{N}$ is given by

$$
\begin{aligned}
\gamma_{2} & \equiv\left(\operatorname{Ai}^{\prime}(0)\right)^{2} \doteq 0.066987484 \\
\gamma_{1} & \equiv\left(\operatorname{Ai}^{\prime}(0)\right)^{2}+\frac{1}{3} \operatorname{Ai}(0) \doteq 0.185330168 \\
\gamma_{4} & \equiv \frac{1}{4}\left(\operatorname{Ai}^{\prime}(0)\right)^{2}-\frac{1}{24} \operatorname{Ai}(0) \doteq 0.001954035
\end{aligned}
$$

for the indicated values of $\beta=2,1,4$, where we have used the formula $K_{\text {Airy }}(t, t)=$ $\left(\operatorname{Ai}^{\prime}(t)\right)^{2}-t(\operatorname{Ai}(t))^{2}$ and $\int_{-\infty}^{0} \operatorname{Ai}(u) d u=\frac{2}{3}, \int_{0}^{\infty} \operatorname{Ai}(u) d u=\frac{1}{3}$ (see $\mathrm{AbSt}$ ). Thus setting $t \rightarrow \hat{t} / \gamma_{\beta}, \beta=2,1,4$, rescales the axis so that the density of the expected number of eigenvalues per unit $\hat{t}$-interval is one.

The distributions $F^{(\beta)}\left(L_{0}\right), \beta=1,2,4$, are the celebrated Tracy-Widom distributions which turn out to have applications in an extraordinary variety of different areas of pure and applied mathematics (see for example the recent review [TW6]). The distributions $F^{(\beta)}\left(L_{0}\right)$ can all be expressed in terms of a certain solution of the Painlevé II equation (TW4, TW5).

The literature on edge scaling, in particular in the physics community, is vast, and we make no attempt to present an exhaustive survey. Rather we will focus on aspects of the literature which are particularly relevant to this paper. In the physics literature, early work on edge scaling for $\beta=2$ is due to Moore $\underline{\mathrm{MO}}$ and Bowick and Brézin $\mathrm{BoBr}$. In the mathematical literature for $\beta=2$ with Gaussian weight $V(x)=x^{2}$, early work can be found in Forrester $[\mathrm{F}]$ and in the seminal work of Tracy and Widom TW4, where the authors derived the Painlevé II representation mentioned above for $F^{(2)}$. For $\beta=1$ and 4 in the Gaussian case $V(x)=x^{2}$, the Painlevé expressions for $F^{(\beta)}$ were obtained by Tracy and Widom in TW5, but without computing directly the edge scaling limit of the Fredholm determinants. The edge scaling limits of matrix kernels $K_{N, \beta}, \beta=1$, 4 , in the Gaussian case were obtained by Forrester, Nagao and Honner in [FNH]. The convergence of the Fredholm determinants in the Gaussian case for $\beta=1,4$ (and also for $\beta=2$ ) was first proved only recently by Tracy and Widom in [TW3.

Universality at the edge for $\beta=2$ was considered by many authors in the physics literature (see e.g. $[\mathrm{KaFr}$ ), and for the cases $\beta=1,4$ see e.g. [SeVe]. The proof of universality at the edge for $\beta=2$ in Theorem 1.1 above is based on the estimates in DKMVZ2 and does not use any results from [W] TW2 DG]. Many researchers have noted that universality at the edge for $\beta=2$ is true (see e.g. $[\mathrm{CKu}$ ), but we believe that the details of the proof (Theorem 1.1 $\beta=2$ ) have not been written down previously. In St1, St2, St3], for $\beta=2,1,4$, Stojanovic proves universality at the edge (and also in the bulk) in the special case of an even quartic (two-interval) potential considered previously by Bleher and Its [BI for $\beta=2$. Stojanovic uses a variant of the formulae in $[\mathrm{W}$ ] together with the asymptotics for OP's obtained in BI]. Universality for the distribution of the largest eigenvalue for a wide class of real and complex Wigner ensembles (see [M1] ) was proven by Soshnikov in [So]: the methods in $[\mathrm{So}$ ] are completely different from those in the present paper and are based on the method of moments. Laguerre ensembles have been considered by many authors, see e.g. [F] [FNH]. Various universality issues at the soft edge, and also at the hard edge and in the bulk, for generalized Laguerre ensembles for $\beta=2$ were analyzed recently in $[\underline{V}$. The authors are currently completing an analysis of 
universality questions for such ensembles in the cases $\beta=1$ and 4 , together with Kriecherbauer and Vanlessen, see DGKV].

We complete this introduction with a description of Widom's result [W] which is basic for our approach in this paper. Widom's method applies to general weights $w_{\beta}$ with the property that $w_{\beta}^{\prime} / w_{\beta}$ is a rational function. This property certainly holds for our weights as in (1.4), (1.2), and also for general Laguerre type weights which we consider in the forthcoming paper DGKV]. Introduce the matrices

$$
D_{N} \equiv\left(\left(D \phi_{j}, \phi_{k}\right)\right)_{0 \leq j, k \leq N-1}, \quad \epsilon_{N} \equiv\left(\left(\epsilon \phi_{j}, \phi_{k}\right)\right)_{0 \leq j, k \leq N-1} .
$$

It follows from [TW1 Section 6] that the matrix $D_{N}$ is banded with bandwidth $2 n+1$ where

$$
n \equiv 2 m-1 .
$$

Thus $\left(D_{N}\right)_{j k}=0$ if $|j-k|>n$. Next, let $N$ be greater than $n$, and introduce the following $N$-dependent $n$-column vectors

$$
\begin{aligned}
\Phi_{1}(x) & \equiv\left(\phi_{N-n}(x), \cdots, \phi_{N-1}(x)\right)^{T} \\
\Phi_{2}(x) & \equiv\left(\phi_{N}(x), \cdots, \phi_{N+n-1}(x)\right)^{T} \\
\epsilon \Phi_{1}(x) & \equiv\left(\epsilon \phi_{N-n}(x), \cdots, \epsilon \phi_{N-1}(x)\right)^{T} \\
\epsilon \Phi_{2}(x) & \equiv\left(\epsilon \phi_{N}(x), \cdots, \epsilon \phi_{N+n-1}(x)\right)^{T}
\end{aligned}
$$

and the following $2 n \times 2 n$ matrices consisting of four $n \times n$ blocks

$$
B \equiv\left(\begin{array}{cc}
B_{11} & B_{12} \\
B_{21} & B_{22}
\end{array}\right)=\left(\left(\epsilon \phi_{j}, \phi_{k}\right)\right)_{N-n \leq j, k \leq N+n-1} .
$$

and

$$
A \equiv\left(\begin{array}{cc}
0 & A_{12} \\
A_{21} & 0
\end{array}\right)=\left(\begin{array}{cc}
0 & D_{12} \\
-D_{21} & 0
\end{array}\right)
$$

where $\left(\begin{array}{cc}D_{11} & D_{12} \\ D_{21} & D_{22}\end{array}\right) \equiv\left(\left(D \phi_{j}, \phi_{k}\right)\right)_{N-n \leq j, k \leq N+n-1}$. Finally, set

$$
C=\left(\begin{array}{cc}
C_{11} & C_{12} \\
C_{21} & C_{22}
\end{array}\right) \equiv\left(\begin{array}{cc}
I_{n}+(B A)_{11} & (B A)_{12} \\
(B A)_{21} & (B A)_{22}
\end{array}\right) .
$$

Note that

$$
C_{11}=I_{n}+B_{12} A_{21}=I_{n}-B_{12} D_{21} .
$$

The main result in [W] is the following pair of formulae for $S_{N, 1}$ and $S_{N / 2,4}$

$$
\begin{aligned}
S_{N, 1}(x, y)=K_{N}(x, y)-\left(\Phi_{1}(x)^{T}, 0^{T}\right) & \cdot\left(A C\left(I_{2 n}-B A C\right)^{-1}\right)^{T} \\
\cdot & \left(\epsilon \Phi_{1}(y)^{T}, \epsilon \Phi_{2}(y)^{T}\right)^{T}
\end{aligned}
$$

and

$$
\begin{aligned}
S_{N / 2,4}(x, y)=K_{N}(x, y) & +\Phi_{2}(x)^{T} \cdot D_{21} \cdot \epsilon \Phi_{1}(y) \\
& +\Phi_{2}(x)^{T} \cdot D_{21} C_{11}^{-1} B_{11} D_{12} \cdot \epsilon \Phi_{2}(y) .
\end{aligned}
$$


Observe that $S_{N, 1}$ and $S_{N / 2,4}$ are sums of the $\beta=2$ kernel $K_{N}(x, y)$ together with correction terms that depend only on $\phi_{N+j}$ for $j \in\{-n, \cdots, n-1\}$. The $\beta=4$ case is different from the case $\beta=1$ since, by (1.18), for any $x \in \mathbb{R}$,

$$
S_{N / 2,4}(x,+\infty)=0, \quad K_{N}(x,+\infty)=0 .
$$

Therefore in (1.43) for any (even) $N$ and for all $x \in \mathbb{R}$

$$
\Phi_{2}(x)^{T} \cdot D_{21} \cdot \epsilon \Phi_{1}(+\infty)+\Phi_{2}(x)^{T} \cdot D_{21} C_{11}^{-1} B_{11} D_{12} \cdot \epsilon \Phi_{2}(+\infty)=0 .
$$

As the entries of $\Phi_{2}(x)$ are functionally independent, and as $D_{12}$ is invertible for large $N$ (see DG, (2.13)]), it follows that

$$
\epsilon \Phi_{1}(+\infty)+C_{11}^{-1} B_{11} D_{12} \cdot \epsilon \Phi_{2}(+\infty)=0
$$

for large $N$. From the definition of $\epsilon$ for any integrable $\psi$

$$
\epsilon \psi(y)=\frac{1}{2} \int_{-\infty}^{\infty} \psi(t) d t-\int_{y}^{\infty} \psi(t) d t=\epsilon \psi(+\infty)-\int_{y}^{\infty} \psi(t) d t .
$$

Hence (1.43), 1.45) imply

$$
\begin{aligned}
S_{N / 2,4}(x, y)=K_{N}(x, y) & +\Phi_{2}(x)^{T} \cdot D_{21} \cdot\left(-\int_{y}^{\infty} \Phi_{1}(t) d t\right) \\
& +\Phi_{2}(x)^{T} \cdot D_{21} C_{11}^{-1} B_{11} D_{12} \cdot\left(-\int_{y}^{\infty} \Phi_{2}(t) d t\right) .
\end{aligned}
$$

Formula (1.48) makes clear the decay properties of $S_{N / 2,4}(x, y)$ as $x, y \rightarrow+\infty$. Note that $S_{N, 1}$ does not satisfy (1.44): this is the reason why we introduce auxiliary functions (cf. $G=\operatorname{diag}\left(g, g^{-1}\right)$ ) when proving convergence of the determinant in Corollary 1.3. As noted earlier, the question of convergence of the determinants for $\beta=1,4$ in the Gaussian case was first treated in [TW3].

The following observations apply to the 21 entries in the matrix kernels in the $\beta=1$ and 4 cases. Note that by (1.17), $\left(\epsilon S_{N, 1}\right)(x, y)$ is skew symmetric. Thus

$$
\left(\epsilon S_{N, 1}\right)(x, y)=\left(\epsilon S_{N, 1}\right)(x, y)-\left(\epsilon S_{N, 1}\right)(y, y)=-\int_{x}^{y} S_{N, 1}(t, y) d t .
$$

Also, from (1.18), we see that $\left(\epsilon S_{N / 2,4}\right)(+\infty, y)=0$ for all $y \in \mathbb{R}$. Together with (1.47), this implies that

$$
\left(\epsilon S_{N / 2,4}\right)(x, y)=-\int_{x}^{\infty} S_{N / 2,4}(t, y) d t .
$$

These observations simplify evaluation of integrals of the CD kernel, and also integrals of the functions $\phi_{N+j}$ in Sections 3 and 4 below.

Remark 1.4. We note that (1.49) is also true for $S_{N / 2,4}$, but (1.50) is more relevant for the calculations that follow.

In Section 2] we prove Theorem 1.1 and Corollary 1.3 using results on the edge scaling limits of the CD terms and the correction terms in $K_{N, 1}$ and $K_{N, 4}$. These scaling limits are proved in turn in Section 3 for the CD terms, and in Section 4 for the correction terms. Note that Corollary 1.2 is an immediate consequence of Theorem 1.1

Notational remark: Throughout this paper $c, c^{\prime}, C, C(m), c_{1}, c_{2}, \cdots$ refer to constants independent of $N, \xi, \eta$. The symbols $c, c^{\prime}, C, \cdots$ refer to generic constants, 
whose precise value may change from one inequality to another. The symbol $c_{N}$ however always refers to the $N$-dependent constant (3.1) below.

Acknowledgments. The work of the first author was supported in part by NSF grants DMS-0296084 and DMS-0500923. The second author would like to thank the Courant Institute, New York University, where he has spent a part of the academic year 2004-05, for hospitality and financial support. The second author also would like to thank Caltech for hospitality and financial support. Finally, the second author would like to thank the Swedish foundation STINT for providing basic support to visit Caltech.

\section{Proofs of Theorem 1.1 and Corollary 1.3}

The key estimates for the proofs of Theorem 1.1 and Corollary 1.3 are obtained below in Section 3 for the CD terms and in Section 4 for the correction terms.

2.1. Proof of Theorem 1.1. Inequality (3.8) proves the result for the $\beta=2$ case.

In the case $\beta=4$, we use 1.48) and consider the CD part and the correction term

separately. The properly scaled 11,22 and 12 entries of $K_{N / 2,4}^{\left(\lambda_{(N)}\right)}\left(\xi^{(N)}, \eta^{(N)}\right)$ converge to the corresponding entries in (1.28) et seq. with the error estimate $o(1) e^{-c \xi} e^{-c \eta}$, uniformly for $\xi, \eta \in\left[L_{0},+\infty\right)$ : this follows from (3.8) for the CD kernel part, and from (4.22) and (4.17), respectively, for the correction term. By (1.50), (3.56) and (4.26), the (unscaled) 21 entry $\left(\epsilon S_{N / 2,4}\right)\left(\xi^{(N)}, \eta^{(N)}\right)$ of $K_{N / 2,4}$ satisfies

$$
\begin{aligned}
\mid 2\left(\epsilon S_{N / 2,4}\right)\left(\xi^{(N)}, \eta^{(N)}\right) & -\left[\left(-\int_{\xi}^{\infty} K_{\text {Airy }}(t, \eta) d t\right.\right. \\
+ & \left.\frac{1}{2}\left(\int_{\xi}^{\infty} \operatorname{Ai}(t) d t\right)\left(\int_{\eta}^{\infty} \operatorname{Ai}(t) d t\right)\right] \mid \leq o(1) e^{-c \xi} e^{-c \eta}
\end{aligned}
$$

uniformly for $\xi, \eta \in\left[L_{0},+\infty\right)$. This completes the proof of Theorem 1.1 for $\beta=4$.

In the case $\beta=1$, we use (1.42) and again consider the CD part and the correction term separately. The properly scaled 11 and 22 entries of $K_{N, 1}^{\left(\lambda_{(N)}\right)}\left(\xi^{(N)}, \eta^{(N)}\right)$ converge to the corresponding entries in (1.27) et seq. with the error estimates $o(1) e^{-c \xi}$ and $o(1) e^{-c \eta}$, respectively, uniformly for $\xi, \eta \in\left[L_{0},+\infty\right)$ : this follows from (3.8) for the CD kernel part (giving rise to a smaller error $o(1) e^{-c \xi} e^{-c \eta}$ ) and from (4.49) for the correction term. The properly scaled 12 entry converges to the corresponding entry in (1.27) et seq. with error $o(1) e^{-c \xi} e^{-c \eta}$, uniformly for $\xi, \eta \in\left[L_{0},+\infty\right)$ : this follows from (3.8) for the CD kernel part and from (4.39) for the correction term. Finally, in view of (1.49), (3.56) and (4.50), the (unscaled) 21 entry of $K_{N, 1}^{\left(\lambda_{(N)}\right)}\left(\xi^{(N)}, \eta^{(N)}\right)$ satisfies

$$
\begin{aligned}
\mid\left(\epsilon S_{N, 1}\right)\left(\xi^{(N)}, \eta^{(N)}\right)-\left[-\int_{\xi}^{\eta} K_{\text {Airy }}(t \eta) d t-\frac{1}{2} \int_{\xi}^{\eta} \operatorname{Ai}(s) d s\right. \\
\left.+\frac{1}{2}\left(\int_{\xi}^{\eta} \operatorname{Ai}(s) d s\right)\left(\int_{\eta}^{\infty} \operatorname{Ai}(t) d t\right)\right] \mid \leq o(1) e^{-c \min (\xi, \eta)}=o(1)
\end{aligned}
$$


with the uniform estimate $o(1)$ for $\xi, \eta \geq L_{0}$. In order to obtain the same form for the limit as claimed in Theorem 1.1 we note that for all $\xi, \eta \in \mathbb{R}$

$$
\begin{aligned}
-\int_{\xi}^{\eta} & K_{\text {Airy }}(t, \eta) d t+\frac{1}{2}\left(\int_{\xi}^{\eta} \operatorname{Ai}(t) d t\right)\left(\int_{\eta}^{\infty} \operatorname{Ai}(t) d t\right) \\
& =-\int_{\xi}^{\infty} K_{\text {Airy }}(t, \eta) d t+\frac{1}{2}\left(\int_{\xi}^{\infty} \operatorname{Ai}(t) d t\right)\left(\int_{\eta}^{\infty} \operatorname{Ai}(t) d t\right) .
\end{aligned}
$$

Indeed, a direct calculation using the representation (1.25) for $K_{\text {Airy }}$ shows that the RHS of (2.3) is skew symmetric in $\xi$ and $\eta$. In particular, the RHS vanishes for $\xi=\eta$, as is also evident for the LHS. But the $\xi$ derivatives of both sides are equal and hence the identity follows. This finishes the proof of Theorem 1.1

2.2. Proof of Corollary 1.3. The following basic fact is well-known (see e.g. [ReSi]). Let $D=d / d x$ denote differentiation and let $\rho(x)$ be any positive function such that $\rho^{-1} \in L^{2}(\mathbb{R})$. Then the operator

$$
A=\frac{1}{\rho} \frac{1}{D+I}
$$

is Hilbert-Schmidt in $L^{2}(\mathbb{R})$. Indeed, by the Fourier transform, $A$ is unitarily equivalent to an operator with square integrable kernel $\widehat{\left(\rho^{-1}\right)}\left(k-k^{\prime}\right) \frac{1}{i k^{\prime}+1}, k, k^{\prime} \in \mathbb{R}$.

2.2.1. The case $\beta=2$. Let $\lambda_{1}$ denote the largest eigenvalue of the matrix $M$ in the unitary ensemble. It is well-known (see e.g. [TW2]) that for finite $N$

$$
\begin{aligned}
\operatorname{Prob}\{ & \left.\lambda_{1} \leq c_{N}\left(1+\frac{L_{0}}{\alpha_{N} N^{2 / 3}}\right)+d_{N}\right\} \\
& =\operatorname{det}\left(1-\left.\frac{c_{N}}{\alpha_{N} N^{2 / 3}} K_{N}\left(\xi^{(N)}, \eta^{(N)}\right)\right|_{L^{2}\left(\left[L_{0},+\infty\right)\right)}\right) .
\end{aligned}
$$

Since $K_{N}$ is finite rank, it is indeed trace class. As the trace class determinant is continuous under the trace class convergence, we only have to prove that

$$
\Delta_{N}(\xi, \eta) \equiv \frac{c_{N}}{\alpha_{N} N^{2 / 3}} K_{N}\left(\xi^{(N)}, \eta^{(N)}\right)-K_{\text {Airy }}(\xi, \eta) \rightarrow 0, \quad \text { as } N \rightarrow \infty,
$$

in the trace norm in $L^{2}\left(\left[L_{0},+\infty\right)\right)$, in order to prove Corollary 1.3 for $\beta=2$. Let $\chi_{L_{0}}^{\#}(\xi)$ be a $C^{\infty}$ function such that $\chi_{L_{0}}^{\#}(\xi)=1$ for $\xi \geq L_{0}$ and $\chi_{L_{0}}^{\#}(\xi)=0$ for $\xi \leq L_{0}-1$. We will show that

$$
\chi_{L_{0}}^{\#} \Delta_{N} \chi_{L_{0}}^{\#} \rightarrow 0, \quad N \rightarrow \infty
$$

in the trace norm in $L^{2}(\mathbb{R})$. But then $\chi_{L_{0}} \Delta_{N} \chi_{L_{0}}=\chi_{L_{0}}\left(\chi_{L_{0}}^{\#} \Delta_{N} \chi_{L_{0}}^{\#}\right) \chi_{L_{0}}$ also converges to zero in trace norm in $L^{2}(\mathbb{R})$, where $\chi_{L_{0}}$ is the characteristic function of $\left[L_{0},+\infty\right)$, and this clearly proves (2.5).

Let $\rho(\xi)=\left(1+\xi^{2}\right)^{1 / 2}$ and write

$$
\chi_{L_{0}}^{\#} \Delta_{N} \chi_{L_{0}}^{\#}=\left[\frac{1}{\rho} \frac{1}{D+I}\right]\left[(D+I) \rho \chi_{L_{0}}^{\#} \Delta_{N} \chi_{L_{0}}^{\#}\right] .
$$

The first operator is Hilbert-Schmidt (see (2.4) ) and the second operator is of order $O\left(N^{-2 / 3}\right)$ in Hilbert-Schmidt norm by (3.8), with $L_{0}$ replaced with $L_{0}-1$. This proves (2.6). 
2.2.2. The case $\beta=4$. Let $\lambda_{1}$ denote the largest eigenvalue of the matrix $M$ in the symplectic ensemble. Then in [TW2] the authors prove

$$
\begin{aligned}
\operatorname{Prob}\left\{\lambda_{1}\right. & \left.\leq c_{N}\left(1+\frac{L_{0}}{\alpha_{N} N^{2 / 3}}\right)+d_{N}\right\} \\
& =\sqrt{\operatorname{det}\left(1-\left.\frac{c_{N}}{\alpha_{N} N^{2 / 3}} K_{N / 2,4}^{\left(\lambda_{(N)}\right)}\left(\xi^{(N)}, \eta^{(N)}\right)\right|_{L^{2}\left(\left[L_{0},+\infty\right)\right)}\right)} .
\end{aligned}
$$

The proof will therefore be complete if we could prove that all the four entries of $K_{N / 2,4}^{\left(\lambda_{(N)}\right)}\left(\xi^{(N)}, \eta^{(N)}\right)$ converge to the corresponding entries of $K^{(4)}(\xi, \eta)$ in trace class norm in $L^{2}\left(\left[L_{0}, \infty\right)\right)$. Again we use (1.48) and prove the trace class convergence of the CD part and of the correction term separately. The trace class convergence of the CD parts of all the four entries of $K_{N / 2,4}^{\left(\lambda_{(N)}\right)}$ follows by using (3.8) and (3.56) together with the trace class convergence method in Subsection 2.2.1

To prove the convergence in trace class for the 11 and 22 correction terms, we must show that

$$
\begin{gathered}
\Delta_{N}(\xi, \eta) \equiv \frac{c_{N}}{\alpha_{N} N^{2 / 3}}\left[\Phi_{2}\left(\xi^{(N)}\right)^{T} \cdot D_{21} \cdot\left(-\int_{\eta^{(N)}}^{\infty} \Phi_{1}(t) d t\right)\right. \\
\quad+\Phi_{2}\left(\xi^{(N)}\right)^{T} \cdot D_{21} C_{11}^{-1} B_{11} D_{12} \cdot\left(-\int_{\eta^{(N)}}^{\infty} \Phi_{2}(t) d t\right) \\
\left.-\left(-\frac{1}{2} \operatorname{Ai}(\xi) \int_{\eta}^{\infty} \operatorname{Ai}(t) d t\right)\right]
\end{gathered}
$$

(cf. (4.18), 4.22) ) converges to zero in trace class in $L^{2}\left(\left[L_{0}, \infty\right)\right)$. But $\Delta_{N}$ is an operator with finite rank at most $n+1=2 m=\operatorname{deg} V$, independent of $N$. For such operators we have the following inequality

$$
\left\|\Delta_{N}\right\|_{1} \leq \sqrt{2 m}\left\|\Delta_{N}\right\|_{H S}
$$

where $\|\cdot\|_{1},\|\cdot\|_{H S}$ denote the trace norm, Hilbert-Schmidt norm in $L^{2}\left(\left[L_{0}, \infty\right)\right)$, respectively. Indeed, $\left|\Delta_{N}\right|=\sqrt{\Delta_{N}^{*} \Delta_{N}}$ is also an operator of rank at most $2 \mathrm{~m}$, and hence it has at most $2 m$ nonzero eigenvalues, $\sigma_{1} \geq \sigma_{2} \geq \cdots \geq \sigma_{j}>0,0 \leq j \leq 2 m$. Thus

$$
\left\|\Delta_{N}\right\|_{1}=\operatorname{tr}\left|\Delta_{N}\right|=\sum_{i=1}^{j} \sigma_{i} \leq \sqrt{j}\left(\sum_{i=1}^{j} \sigma_{i}^{2}\right)^{1 / 2} \leq \sqrt{2 m}\left\|\Delta_{N}\right\|_{H S} .
$$

But from (4.22), $\left\|\Delta_{N}\right\|_{H S}=o(1)\left(\int_{L_{0}}^{\infty} \int_{L_{0}}^{\infty} e^{-c \xi} e^{-c \eta} d \xi d \eta\right)^{1 / 2}=o(1), N \rightarrow \infty$, and we conclude that $\left\|\Delta_{N}\right\|_{1} \rightarrow 0, N \rightarrow \infty$, as desired. A similar argument using (4.17) for the 12 entry and (4.26) for the 21 entry, completes the proof of Corollary 1.3 for $\beta=4$.

2.2.3. The case $\beta=1$. Let $\lambda_{1}$ denote the largest eigenvalue of the matrix $M$ in the orthogonal ensemble. Let $g(\xi)=\sqrt{1+\xi^{2}}$ and set $G(\xi)=\left(\begin{array}{cc}g(\xi) & 0 \\ & \\ 0 & g^{-1}(\xi)\end{array}\right)$. Note that $g^{-1}(\xi) \in L^{2}(\mathbb{R})$. Let $g_{(N)}(t)=\sqrt{1+\left[\frac{\alpha_{N} N^{2 / 3}}{c_{N}}\left(t-c_{N}-d_{N}\right)\right]^{2}}$ and $G_{(N)}(\xi)=$ $\left(\begin{array}{cc}g_{(N)}(\xi) & 0 \\ 0 & g_{(N)}^{-1}(\xi)\end{array}\right)$. Note that $g_{(N)}\left(\xi^{(N)}\right)=g(\xi)$. Recall the definition of $\operatorname{det}_{2}$ 
in the Introduction. A slight modification of the calculations in [TW2, Section 9] shows that

$$
\begin{aligned}
\operatorname{Prob}\{ & \left.\lambda_{1} \leq c_{N}\left(1+\frac{L_{0}}{\alpha_{N} N^{2 / 3}}\right)+d_{N}\right\} \\
& =\sqrt{\operatorname{det}_{2}\left(1-\left.\frac{c_{N}}{\alpha_{N} N^{2 / 3}}\left(G_{(N)} K_{N / 2,4}^{\left(\lambda_{(N)}\right)} G_{(N)}^{-1}\right)\left(\xi^{(N)}, \eta^{(N)}\right)\right|_{L^{2}\left(\left[L_{0},+\infty\right)\right)}\right)} .
\end{aligned}
$$

In [TW2, Section 9] the authors use the fact that $\operatorname{det}(1+A B)=\operatorname{det}(1+B A)$ for appropriate operators $A$ and $B$. But one clearly has the freedom to write $A B=A G_{(N)}^{-1} G_{(N)} B$, and so we also have $\operatorname{det}(1+A B)=\operatorname{det}\left(1+A G_{(N)}^{-1} G_{(N)} B\right)=$ $\operatorname{det}\left(1+G_{(N)} B A G_{(N)}^{-1}\right)$ and this leads to (2.8). We have chosen $G_{(N)}$ as above in such a way as to ensure that $1+G_{(N)} B A G_{(N)}^{-1}$ has a 2-determinant, but there is clearly great freedom in the choice of $g_{(N)}$, and hence of $G_{(N)}$. From (2.8) we see that in order to prove (1.33) it is enough to show Si] that the diagonal (respectively the off-diagonal) entries of $\frac{c_{N}}{\alpha_{N} N^{2 / 3}}\left(G_{(N)} K_{N / 2,4}^{\left(\lambda_{(N)}\right)} G_{(N)}^{-1}\right)\left(\xi^{(N)}, \eta^{(N)}\right)$ converge to the respective entries of $\left(G K^{(1)} G^{-1}\right)(\xi, \eta)$ in trace (respectively Hilbert-Schmidt) norm in $L^{2}\left(\left[L_{0}, \infty\right)\right)$.

We consider first the 11 entry (again the 22 entry can be considered similarly). This entry has the form

$\frac{c_{N}}{\alpha_{N} N^{2 / 3}} g_{(N)}\left(\xi^{(N)}\right) S_{N, 1}\left(\xi^{(N)}, \eta^{(N)}\right) g_{(N)}^{-1}\left(\eta^{(N)}\right)=\frac{c_{N}}{\alpha_{N} N^{2 / 3}} g(\xi) S_{N, 1}\left(\xi^{(N)}, \eta^{(N)}\right) g^{-1}(\eta)$

where $S_{N, 1}$ is given by the CD part and the correction term as in (1.42). The proof that $g(\xi)\left[\frac{c_{N}}{\alpha_{N} N^{2 / 3}} K_{N}\left(\xi^{(N)}, \eta^{(N)}\right)-K_{\text {Airy }}(\xi, \eta)\right] g^{-1}(\eta) \rightarrow 0, N \rightarrow \infty$, in trace norm in $L^{2}\left(\left[L_{0}, \infty\right)\right)$ is completely analogous to the $\beta=2$ case in Subsection 2.2.1] (note that $g$ and its derivative are polynomially bounded) and the details are left to the reader.

As in the $\beta=4$ case above, the fact that the correction term in the 11 entry has a fixed maximal rank independent of $N$ implies that the trace norm convergence follows from the Hilbert-Schmidt convergence. But by (4.40), 4.49)

$$
\begin{aligned}
\mid g(\xi)\left[-\Phi_{1}\left(\xi^{(N)}\right)^{T} \cdot G_{11} \cdot\left(-\int_{\eta^{(N)}}^{\infty} \Phi_{1}(t) d t\right)-\Phi_{1}\left(\xi^{(N)}\right)^{T} \cdot G_{12} \cdot\left(-\int_{\eta^{(N)}}^{\infty} \Phi_{2}(t) d t\right)\right. \\
-\Phi_{1}\left(\xi^{(N)}\right)^{T} \cdot G_{11} \cdot \epsilon \Phi_{1}(+\infty)-\Phi_{1}\left(\xi^{(N)}\right)^{T} \cdot G_{12} \cdot \epsilon \Phi_{2}(+\infty) \\
\left.-\frac{1}{2} \operatorname{Ai}(\xi) \int_{-\infty}^{\eta} \operatorname{Ai}(t) d t\right] g^{-1}(\eta) \mid \leq o(1) g(\xi) e^{-c \xi} g^{-1}(\eta)
\end{aligned}
$$

which is $o(1)$ in Hilbert-Schmidt norm in $L^{2}\left(\left[L_{0}, \infty\right)\right)$. This proves the trace class convergence of the 11 (and similarly of the 22) entry.

Finally, we note from the uniform pointwise bounds in (1.29) that the error terms in the 12 and 21 entries are bounded by $o(1) g(\xi) e^{-c \xi} e^{-c \eta} g(\eta)$ and $o(1) g^{-1}(\xi) g^{-1}(\eta)$, respectively, uniformly for $\xi, \eta \geq L_{0}$. This immediately implies the Hilbert-Schmidt convergence of the off-diagonal entries to their appropriate limits. This completes the proof of Corollary 1.3

Remark 2.1. With a little more work one can show that in the $\beta=1$ case the off-diagonal entries (apart from the term $g^{-1}(\xi) \operatorname{sgn}(\xi-\eta) g^{-1}(\eta)$ ) in fact converge in trace class norm, and not just in Hilbert-Schmidt norm. 
Remark 2.2. As noted earlier, there is considerable freedom in the choice of the auxiliary function $g$. We see that all we need is that $g, g^{\prime}$ are polynomially bounded and $g^{-1} \in L^{2}(\mathbb{R})$.

\section{The edge scaling limits of the Christoffel-Darboux $(\beta=2)$} KERNEL, AND OF ITS DERIVATIVES AND INTEGRALS

3.1. Auxiliary facts from DKMVZ2. We now recall some notation from [ibid.]. Let $d \mu_{N}^{(\text {eq })}(x)$ denote the equilibrium measure (see e.g. [SaTo $)$ for OP's corresponding to the rescaled weight $e^{-N V_{N}(x)}, V_{N}=\frac{1}{N} V\left(c_{N} x+d_{N}\right)$, where $c_{N}, d_{N}$ are the so-called Mhaskar-Rakhmanov-Saff (MRS) numbers (see MhSa, Ra). For $V(x)=\kappa_{2 m} x^{2 m}+\kappa_{2 m-1} x^{2 m-1}+\cdots$ as in (1.19), we have [ibid., Thm. 2.1] to any order $q$ as $N \rightarrow \infty$

$$
c_{N}=\left(\frac{1}{\kappa_{2 m}} \frac{(2 m) ! !}{m(2 m-1) ! !}\right)^{1 /(2 m)} N^{1 /(2 m)}+\sum_{j=0}^{q} c_{(j)} N^{-j /(2 m)}+O\left(N^{-(q+1) /(2 m)}\right)
$$

and

$$
d_{N}=-\frac{\kappa_{2 m-1}}{2 m \kappa_{2 m}}+\sum_{j=1}^{q} d_{(j)} N^{-j /(2 m)}+O\left(N^{-(q+1) /(2 m)}\right) .
$$

As $N \rightarrow \infty$, the equilibribum measure is absolutely continuous with respect to Lebesgue measure, $d \mu_{N}^{(\mathrm{eq})}(x)=\psi_{N}^{(\mathrm{eq})}(x) d x$, and is supported on the (single) interval $[-1,1]$,

$$
\psi_{N}^{(\mathrm{eq})}(x) \equiv \psi_{N}(x)=\frac{1}{2 \pi}\left|1-x^{2}\right|^{1 / 2} \chi_{[-1,1]}(x) h_{N}(x)
$$

(see [ibid., (2.4)]) where $h_{N}(x)$ is a real polynomial of degree $2 m-2$ satisfying [ibid., Prop. 5.3]

$$
h_{N}(x) \geq h_{\min }>0, \quad x \in \mathbb{R}, \quad N \geq N_{1}(V) .
$$

Set [ibid., (5.33)]

$$
g(z) \equiv g_{N}(z)=\int_{-1}^{1} \log (z-x) d \mu_{N}^{(\mathrm{eq})}(x)=\int_{-1}^{1} \log (z-x) \frac{1}{2 \pi}\left|1-x^{2}\right|^{1 / 2} h_{N}(x) d x,
$$

$z \in \mathbb{C} \backslash(-\infty,-1]$, and for $z \in(-1,1)$ [ibid., (5.34)]

$$
\Xi_{N}(z) \equiv g_{+}(z)-g_{-}(z)=i \int_{z}^{1}\left|1-x^{2}\right|^{1 / 2} h_{N}(x) d x .
$$

We also use the same symbol for the analytic continuation of $\Xi_{N}$ to $\mathbb{C} \backslash((-\infty,-1] \cup$ $[1,+\infty))$.

Notational remark: Here we denote by $\Xi_{N}$ what was denoted by $\xi_{N}$ in [ibid.].

For a fixed $\delta>0$ sufficiently small (cf. [DG] Rem. 4.3]), let $R$ denote the matrix function defined in DKMVZ2, (7.47)]. The function $R$ is analytic in the complement of the contour $\hat{\Sigma}_{R}$ as in [ibid., Fig. 7.6] and is continuous up to the boundary. Furthermore by [ibid., Thm. 7.10], it has an asymptotic expansion

$$
R(z) \sim I+N^{-1} \sum_{k=0}^{\infty} r_{k}(z) N^{-k /(2 m)}
$$


where $\left\{r_{k}(z)\right\}$ are bounded functions that are analytic in the complement of $\{\mid z-$ $1 \mid=\delta\} \cup\{|z+1|=\delta\}$. The expansion (3.6) is uniform for $z \in \mathbb{C} \backslash \hat{\Sigma}_{R}$. Moreover, by the proof of [ibid., Thm 7.10] and Cauchy's theorem, it follows that (3.6) can be differentiated term by term,

$$
\frac{d^{j}}{d z^{j}} R(z) \sim N^{-1} \sum_{k=0}^{\infty} \frac{d^{j}}{d z^{j}} r_{k}(z) N^{-k /(2 m)}, \quad j=1,2, \cdots,
$$

where again the expansion is uniform for $z \in \mathbb{C} \backslash \hat{\Sigma}_{R}$. Also, each $\frac{d^{j}}{d z^{j}} r_{k}(z)$ is bounded (and analytic) in the complement of $\{|z-1|=\delta\} \cup\{|z+1|=\delta\}$.

3.2. Estimates on the CD kernel and its derivatives. We will only consider the end point 1 (the end point -1 can be considered similarly). Let $L_{0} \in \mathbb{R}$ be fixed. Recall the notation in 1.25), 1.23) and (1.5). Our goal in this Subsection is to prove that for $j, k=0,1$, and some $C=C\left(L_{0}\right)>0, c=c\left(L_{0}\right)>0$, one has uniformly for $\xi, \eta \in\left[L_{0},+\infty\right)$

$$
\left|\partial_{\xi}^{j} \partial_{\eta}^{k}\left[\frac{c_{N}}{\alpha_{N} N^{2 / 3}} K_{N}\left(\xi^{(N)}, \eta^{(N)}\right)-K_{\text {Airy }}(\xi, \eta)\right]\right| \leq C N^{-2 / 3} e^{-c \xi} e^{-c \eta} .
$$

Note that $\mathrm{AbSt}$

$$
\begin{aligned}
& |\operatorname{Ai}(\xi)| \leq C(1+|\xi|)^{-1 / 4}, \quad\left|\operatorname{Ai}^{\prime}(\xi)\right| \leq C(1+|\xi|)^{1 / 4}, \quad \xi \in \mathbb{R}, \\
& \left|d^{q} \operatorname{Ai}(\xi) / d \xi^{q}\right| \leq C_{1} e^{-\xi} \leq C_{2}, \quad \xi \in\left[L_{0},+\infty\right), \quad q=0,1,2, \cdots .
\end{aligned}
$$

3.2.1. Auxiliary notation. Set (see [DKMVZ2, (2.15)] and also [DG, (4.10)])

$$
f_{N}(x)=\alpha_{N} N^{2 / 3}(x-1) \hat{f}_{N}(x)
$$

which satisfies the following (see (the proof of) [DKMVZ2, Proposition 7.3])

(1) $\hat{f}_{N}(x)$ is real analytic on $(1-2 \delta, 1+2 \delta)$, and to any order $q=0,1,2, \cdots$

$$
\hat{f}_{N}(x)=\sum_{j=0}^{q} N^{-j /(2 m)} \hat{f}_{(j)}(x)+O\left(N^{-(q+1) /(2 m)}\right)
$$

uniformly for $x$ in the interval. Moreover, the functions $\hat{f}_{(j)}(x)$ are also real analytic on $1-2 \delta<x<1+2 \delta$

(2) to any order $q=1,2, \cdots$

$$
\alpha_{N} \equiv\left(h_{N}^{2}(1) / 2\right)^{1 / 3}=2 m^{2 / 3}+\sum_{j=1}^{q} N^{-j /(2 m)} \alpha_{(j)}+O\left(N^{-(q+1) /(2 m)}\right)
$$

(3) $f_{N}^{\prime}(x)=-\alpha_{N} N^{2 / 3} W_{N}(x)$, where $W_{N}(x)=\hat{f}_{N}(x)+(x-1) \hat{f}_{N}^{\prime}(x)$ also has an expansion uniform in $x$ to any order $q=0,1,2, \cdots$ as above

$$
W_{N}(x)=\sum_{j=0}^{q} N^{-j /(2 m)} W_{(j)}(x)+O\left(N^{-(q+1) /(2 m)}\right) .
$$

The terms $W_{(j)}(x)$ are real analytic on $1-2 \delta<x<1+2 \delta$

(4) $\max _{k=0,1,2} \max _{1-2 \delta \leq x \leq 1+2 \delta}\left|d^{k} \hat{f}_{N}(x) / d x^{k}\right| \leq M<\infty$ for $N \geq N_{2}(V)$

(5) $\hat{f}_{N}(1)=1=W_{N}(1)$ and $\min _{1-2 \delta \leq x \leq 1+2 \delta} \hat{f}_{N}(x) \geq \frac{1}{2}$ for $N \geq N_{2}(V)$. Also $\hat{f}_{(0)}(1)=1=W_{(0)}(1)$. 
Denote

$$
\begin{array}{ll}
\xi_{N} \equiv \xi /\left(\alpha_{N} N^{2 / 3}\right), & \eta_{N} \equiv \eta /\left(\alpha_{N} N^{2 / 3}\right) \\
I_{N} \equiv\left[L_{0}, \alpha_{N} N^{2 / 3} \delta\right], & I I_{N} \equiv\left[\alpha_{N} N^{2 / 3} \delta,+\infty\right) .
\end{array}
$$

Thus, recalling (1.23),$\xi^{(N)}=c_{N}\left(1+\xi_{N}\right)+d_{N}$ and similarly $\eta^{(N)}=c_{N}\left(1+\eta_{N}\right)+d_{N}$. As above, let $\delta>0$ be fixed and sufficiently small. Consider first $\xi_{N}, \eta_{N}$ in a neighborhood of 0 . Set

$$
\begin{aligned}
g_{N}(\xi) & \equiv \xi \hat{f}_{N}\left(1+\xi_{N}\right) \\
\hat{F}_{N}\left(1+\xi_{N}\right) & \equiv\left(2+\xi_{N}\right)^{1 / 4} \cdot\left(\hat{f}_{N}\left(1+\xi_{N}\right)\right)^{1 / 4} \\
F_{N}\left(1+\xi_{N}\right) & \equiv N^{1 / 6} \alpha_{N}^{1 / 4} \hat{F}_{N}\left(1+\xi_{N}\right)
\end{aligned}
$$

and also

$$
\begin{aligned}
& A_{0}(\xi) \equiv N^{1 / 6} \alpha_{N}^{1 / 4} \hat{F}_{N}\left(1+\xi_{N}\right) \cdot \operatorname{Ai}\left(g_{N}(\xi)\right) \\
& A_{1}(\xi) \equiv N^{-1 / 6} \alpha_{N}^{-1 / 4}\left(\hat{F}_{N}\left(1+\xi_{N}\right)\right)^{-1} \cdot \operatorname{Ai}^{\prime}\left(g_{N}(\xi)\right) .
\end{aligned}
$$

Note that in view of (3.10) (1)(5) and the formula

$$
g_{N}^{\prime}(\xi)=\hat{f}_{N}\left(1+\xi_{N}\right)+\xi_{N} \hat{f}_{N}^{\prime}\left(1+\xi_{N}\right)
$$

there exist $c_{2}>c_{1}>0$ such that

$$
c_{1} \leq \frac{g_{N}(\xi)}{\xi} \leq c_{2}, \quad \xi \in I_{N}
$$

and

$$
c_{1} \leq g_{N}^{\prime}(\xi) \leq c_{2}, \quad\left|g_{N}^{\prime \prime}(\xi)\right| \leq c_{2} N^{-2 / 3}, \quad \xi \in I_{N} .
$$

Similarly one has uniformly for $\xi \in I_{N}$

$$
c_{1} \leq \hat{F}_{N}\left(1+\xi_{N}\right) \leq c_{2}, \quad\left|\frac{d^{k}}{d z^{k}} \hat{F}_{N}(z)\right|_{z=1+\xi_{N}} \mid \leq C(k)
$$

for some $C(k), k=1,2, \cdots$.

3.2.2. Estimates for $(\xi, \eta) \in I_{N} \times I_{N}$. With the above notation the following holds.

Proposition 3.1. For $(\xi, \eta) \in I_{N} \times I_{N}$

$$
\frac{c_{N}}{\alpha_{N} N^{2 / 3}} K_{N}\left(\xi^{(N)}, \eta^{(N)}\right)=K_{\text {Airy }}(\xi, \eta)+\frac{1}{\alpha_{N} N^{2 / 3}} \sum_{j=1}^{4} Q_{1, j}(\xi, \eta)
$$

where

$$
\begin{aligned}
Q_{1,1}(\xi, \eta) \equiv & -\left(\begin{array}{ll}
A_{0}(\eta) & A_{1}(\eta)
\end{array}\right) \cdot\left(\begin{array}{cc}
1 & -i \\
-1 & -i
\end{array}\right) \\
& \cdot \int_{0}^{1}\left(R^{T}\right)^{\prime}\left(1+\xi_{N}+t\left(\eta_{N}-\xi_{N}\right)\right) d t \\
& \cdot\left(\begin{array}{cc}
1 & -i \\
-1 & -i
\end{array}\right)^{-1}\left(\begin{array}{c}
-A_{1}(\xi) \\
A_{0}(\xi)
\end{array}\right)
\end{aligned}
$$


and

$$
\begin{aligned}
Q_{1,2}(\xi, \eta) & \equiv \operatorname{Ai}\left(g_{N}(\xi)\right) \operatorname{Ai}^{\prime}\left(g_{N}(\eta)\right) \cdot T_{N}(\xi, \eta)-\operatorname{Ai}\left(g_{N}(\eta)\right) \operatorname{Ai}^{\prime}\left(g_{N}(\xi)\right) \cdot T_{N}(\eta, \xi) \\
T_{N}(\xi, \eta) & \equiv \frac{\int_{0}^{1} \hat{F}_{N}^{\prime}\left(1+\eta_{N}+\tau\left(\xi_{N}-\eta_{N}\right)\right) d \tau}{\hat{F}_{N}\left(1+\eta_{N}\right)}
\end{aligned}
$$

and

$$
\begin{aligned}
& Q_{1,3}(\xi, \eta) \equiv E_{N}(\xi, \eta) \int_{0}^{\infty} \operatorname{Ai}\left(z+g_{N}(\xi)\right) \operatorname{Ai}\left(z+g_{N}(\eta)\right) d z \\
& E_{N}(\xi, \eta) \equiv \int_{0}^{1}[\eta+\tau(\xi-\eta)]\left[\hat{f}_{N}^{\prime}\left(1+\eta_{N}+\tau\left(\xi_{N}-\eta_{N}\right)\right)\right. \\
&\left.+\int_{0}^{1} \hat{f}_{N}^{\prime}\left(1+\sigma\left(\eta_{N}+\tau\left(\xi_{N}-\eta_{N}\right)\right)\right) d \sigma\right] d \tau
\end{aligned}
$$

and

$$
\begin{aligned}
Q_{1,4}(\xi, \eta) \equiv & \xi^{2} L_{N}(\xi) \int_{0}^{\infty} U_{N}(\xi, z) \operatorname{Ai}\left(z+g_{N}(\eta)\right) d z \\
& +\eta^{2} L_{N}(\eta) \int_{0}^{\infty} \operatorname{Ai}(z+\xi) U_{N}(\eta, z) d z \\
L_{N}(\xi) \equiv & \int_{0}^{1} \hat{f}_{N}^{\prime}\left(1+\sigma \xi_{N}\right) d \sigma \\
U_{N}(\xi, z) \equiv & \int_{0}^{1} \operatorname{Ai}^{\prime}\left(z+\xi+\tau\left(g_{N}(\xi)-\xi\right)\right) d \tau .
\end{aligned}
$$

Proof. First, some algebra: let $Y$ solve the Fokas-Its-Kitaev Riemann-Hilbert problem for the polynomials orthogonal with respect to the weight $e^{-V(x)} d x$ (see DKMVZ2, Thm. 3.1]). Then as in [DKMVZ1 (6.3)] we find for any $x, y \in \mathbb{R}$

$$
\begin{aligned}
K_{N}(x, y) & =e^{-(V(x)+V(y)) / 2} \frac{Y_{11}(y) Y_{21}(x)-Y_{11}(x) Y_{21}(y)}{2 \pi i(x-y)} \\
& =-e^{-(V(x)+V(y)) / 2} \frac{\left(\begin{array}{ll}
1 & 0
\end{array}\right) \cdot Y_{+}^{T}(y) \cdot Y_{+}^{-T}(x) \cdot\left(\begin{array}{ll}
0 & 1
\end{array}\right)^{T}}{2 \pi i(x-y)} .
\end{aligned}
$$

Here and below $+/$ - refer to the boundary values taken from above/below the real axis. (The choice $Y_{+}$is made only for definiteness. Formula (3.23) clearly remains true if $Y_{+}$is replaced with $Y_{-}$.) Consider first $z=1+\xi_{N} \in(1-\delta, 1]$ for $\xi \in\left(-\delta \alpha_{N} N^{2 / 3}, 0\right]$. By DKMVZ2 (4.2), (4.6), (4.22)] we have for $S$, the solution of the Riemann-Hilbert problem [ibid., (4.24)-(4.26)], (cf. [ibid., (7.46),(7.47)])

$$
\begin{aligned}
S_{+}(z)= & c_{N}^{-N \sigma_{3}} e^{-\frac{N l}{2} \sigma_{3}} Y_{+}\left(c_{N} z+d_{N}\right) \\
& \times e^{-N\left(g_{+}(z)-\frac{l}{2}\right) \sigma_{3}}\left(\begin{array}{cc}
1 & 0 \\
-e^{-N\left(g_{+}(z)-g_{-}(z)\right)} & 1
\end{array}\right),
\end{aligned}
$$


where $\sigma_{3}=\left(\begin{array}{cc}1 & 0 \\ 0 & -1\end{array}\right)$ and the constant $l \equiv l_{N}$ is given by [ibid., (5.35)]. Solving for $Y_{+}$and substituting in (3.23) we find for $\xi, \eta \in\left(-\delta \alpha_{N} N^{2 / 3}, 0\right]$

$$
\begin{aligned}
\frac{c_{N}}{\alpha_{N} N^{2 / 3}} & K_{N}\left(\xi^{(N)}, \eta^{(N)}\right)=-\frac{e^{-\frac{N}{2}\left(V_{N}\left(1+\xi_{N}\right)+V_{N}\left(1+\eta_{N}\right)\right)}}{2 \pi i(\xi-\eta)} \\
& \times\left(e^{N\left(g_{+}\left(1+\eta_{N}\right)-\frac{l}{2}\right)} e^{N\left(g_{-}\left(1+\eta_{N}\right)-\frac{l}{2}\right)}\right) \cdot S_{+}^{T}\left(1+\eta_{N}\right) \\
& \times S_{+}^{-T}\left(1+\xi_{N}\right) \cdot\left(\begin{array}{c}
-e^{N\left(g_{-}\left(1+\xi_{N}\right)-\frac{l}{2}\right)} \\
e^{N\left(g_{+}\left(1+\xi_{N}\right)-\frac{l}{2}\right)}
\end{array}\right) \cdot
\end{aligned}
$$

Now note that for $z \in\left(1-\delta, 1\right.$ ], by [ibid., (7.46), (7.47)], $S(z)=R(z) P_{N}(z)$. By [ibid., (7.24), (7.9), (7.23), (7.4)],

$$
\begin{aligned}
P_{N,+}(z)=\sqrt{\pi} e^{i \pi / 6} & \left(\begin{array}{cc}
1 & -1 \\
-i & -i
\end{array}\right)\left(\begin{array}{cc}
F_{N}(z) & 0 \\
0 & 1 / F_{N}(z)
\end{array}\right) \\
& \times A I_{+}\left(f_{N}(z)\right) e^{-i \pi \sigma_{3} / 6}\left(\begin{array}{cc}
1 & 0 \\
-1 & 1
\end{array}\right) e^{N \Xi_{N}(z) \sigma_{3} / 2}
\end{aligned}
$$

where

$$
A I\left(f_{N}(z)\right) \equiv\left(\begin{array}{cc}
\operatorname{Ai}\left(f_{N}(z)\right) & \operatorname{Ai}\left(\omega^{2} f_{N}(z)\right) \\
\operatorname{Ai}^{\prime}\left(f_{N}(z)\right) & \omega^{2} \operatorname{Ai}^{\prime}\left(\omega^{2} f_{N}(z)\right)
\end{array}\right), \quad \omega=e^{2 \pi i / 3} .
$$

For $z \in(-1,1)$, in view of [ibid., (5.38)]

$$
-V_{N}(z)+g_{+}(z)+g_{-}(z)-l=0
$$

and we find from (3.25)

(3.26)

$$
\begin{aligned}
\frac{c_{N}}{\alpha_{N} N^{2 / 3}} & K_{N}\left(\xi^{(N)}, \eta^{(N)}\right)=-\frac{e^{-\pi i / 3}}{2 \pi i(\xi-\eta)}\left(\begin{array}{ll}
1 & 0
\end{array}\right) \cdot A I_{+}^{T}\left(f_{N}\left(1+\eta_{N}\right)\right) \\
& \times\left(\begin{array}{cc}
F_{N}\left(1+\eta_{N}\right) & 0 \\
0 & 1 / F_{N}\left(1+\eta_{N}\right)
\end{array}\right)\left(\begin{array}{cc}
1 & -i \\
-1 & -i
\end{array}\right) R_{+}^{T}\left(1+\eta_{N}\right) \\
& \times R_{+}^{-T}\left(1+\xi_{N}\right)\left(\begin{array}{cc}
1 & -i \\
-1 & -i
\end{array}\right)\left(\begin{array}{cc}
1 / F_{N}\left(1+\xi_{N}\right) & 0 \\
0 & F_{N}\left(1+\xi_{N}\right)
\end{array}\right) \\
& \times A I_{+}^{-T}\left(f_{N}\left(1+\xi_{N}\right)\right) \cdot\left(\begin{array}{c}
0 \\
1
\end{array}\right), \quad \xi, \eta \in\left(-\delta \alpha_{N} N^{2 / 3}, 0\right] .
\end{aligned}
$$

Similar calculations for $z \in[1,1+\delta)$ lead to the same formula for all other cases $\xi<0, \eta>0$, etc., $|\xi|,|\eta| \leq \delta \alpha_{N} N^{2 / 3}$.

Now writing

$$
R^{T}\left(1+\eta_{N}\right)=R^{T}\left(1+\xi_{N}\right)+\left(\eta_{N}-\xi_{N}\right) \int_{0}^{1}\left(R^{T}\right)^{\prime}\left(1+\xi_{N}+t\left(\eta_{N}-\xi_{N}\right)\right) d t
$$


and taking into account $\operatorname{det} A I_{+}\left(f_{N}\left(1+\xi_{N}\right)\right)=-1 /\left(2 \pi i e^{i \pi / 3}\right)$ (use [ibid., (8.38)]) we obtain from (3.26) that

$$
\begin{aligned}
\frac{c_{N}}{\alpha_{N} N^{2 / 3}} & K_{N}\left(\xi^{(N)}, \eta^{(N)}\right)=\frac{1}{\alpha_{N} N^{2 / 3}} Q_{1,1}(\xi, \eta) \\
& +\frac{1}{\xi-\eta}\left\{\operatorname{Ai}\left(g_{N}(\xi)\right) \operatorname{Ai}^{\prime}\left(g_{N}(\eta)\right) \cdot \frac{F_{N}\left(1+\xi_{N}\right)}{F_{N}\left(1+\eta_{N}\right)}-(\xi \leftrightarrow \eta)\right\}
\end{aligned}
$$

where $Q_{1,1}$ is as in (3.19). Now

$$
\begin{aligned}
\frac{F_{N}\left(1+\xi_{N}\right)}{F_{N}\left(1+\eta_{N}\right)} & =\frac{\hat{F}_{N}\left(1+\xi_{N}\right)}{\hat{F}_{N}\left(1+\eta_{N}\right)} \\
& =1+\left(\xi_{N}-\eta_{N}\right) \frac{\int_{0}^{1} \hat{F}_{N}^{\prime}\left(1+\eta_{N}+t\left(\xi_{N}-\eta_{N}\right)\right) d t}{\hat{F}_{N}\left(1+\eta_{N}\right)}
\end{aligned}
$$

and hence using (1.25) we rewrite (3.28) as

$$
\begin{aligned}
\frac{c_{N}}{\alpha_{N} N^{2 / 3}} K_{N}\left(\xi^{(N)}, \eta^{(N)}\right)= & \frac{g_{N}(\xi)-g_{N}(\eta)}{\xi-\eta} K_{\text {Airy }}\left(g_{N}(\xi), g_{N}(\eta)\right) \\
& +\frac{1}{\alpha_{N} N^{2 / 3}}\left(Q_{1,1}(\xi, \eta)+Q_{1,2}(\xi, \eta)\right)
\end{aligned}
$$

where $Q_{1,2}$ is as in (3.20). Next we write $\frac{g_{N}(\xi)-g_{N}(\eta)}{\xi-\eta}=\int_{0}^{1} g_{N}^{\prime}(\eta+\tau(\xi-\eta)) d \tau$, and use (3.14) and

$\hat{f}_{N}\left(1+\eta_{N}+\tau\left(\xi_{N}-\eta_{N}\right)\right)=\hat{f}_{N}(1)+\left(\eta_{N}+\tau\left(\xi_{N}-\eta_{N}\right)\right) \int_{0}^{1} \hat{f}_{N}^{\prime}\left(1+\sigma\left(\eta_{N}+\tau\left(\xi_{N}-\eta_{N}\right)\right)\right) d \sigma$ to conclude that $\frac{g_{N}(\xi)-g_{N}(\eta)}{\xi-\eta}=1+E_{N}(\xi, \eta)$ from (3.21). Hence recalling (1.25) we obtain from (3.30)

$$
\frac{c_{N}}{\alpha_{N} N^{2 / 3}} K_{N}\left(\xi^{(N)}, \eta^{(N)}\right)=K_{\text {Airy }}\left(g_{N}(\xi), g_{N}(\eta)\right)+\frac{1}{\alpha_{N} N^{2 / 3}} \sum_{j=1}^{3} Q_{1, j}(\xi, \eta)
$$

where $Q_{1,3}$ is as in (3.21). Finally again using (1.25) we find

$$
\begin{aligned}
K_{\text {Airy }}\left(g_{N}(\xi), g_{N}(\eta)\right)= & \int_{0}^{\infty} \operatorname{Ai}\left(z+g_{N}(\xi)\right) \operatorname{Ai}\left(z+g_{N}(\eta)\right) d z \\
= & \int_{0}^{\infty} \operatorname{Ai}(z+\xi) \operatorname{Ai}(z+\eta) d z \\
& +\int_{0}^{\infty} \operatorname{Ai}(z+\xi)\left[\operatorname{Ai}\left(z+g_{N}(\eta)\right)-\operatorname{Ai}(z+\eta) d z\right. \\
& +\int_{0}^{\infty}\left[\operatorname{Ai}\left(z+g_{N}(\xi)\right)-\operatorname{Ai}(z+\xi)\right] \operatorname{Ai}\left(z+g_{N}(\eta)\right) d z .
\end{aligned}
$$

The first integral equals $K_{\text {Airy }}(\xi, \eta)$. To evaluate the third integral we recall (3.12), (3.22) and note that

$$
\begin{aligned}
g_{N}(\xi)-\xi & =\xi\left[\hat{f}_{N}\left(1+\xi_{N}\right)-\hat{f}_{N}(1)\right] \\
& =\frac{\xi^{2}}{\alpha_{N} N^{2 / 3}} \cdot \int_{0}^{1} \hat{f}_{N}^{\prime}\left(1+\sigma \xi_{N}\right) d \sigma=\frac{\xi^{2}}{\alpha_{N} N^{2 / 3}} L_{N}(\xi)
\end{aligned}
$$


which implies

$$
\begin{aligned}
\operatorname{Ai}\left(z+g_{N}(\xi)\right)-\operatorname{Ai}(z+\xi)= & \frac{\xi^{2}}{\alpha_{N} N^{2 / 3}} \cdot \int_{0}^{1} \hat{f}_{N}^{\prime}\left(1+\sigma \xi_{N}\right) d \sigma \\
& \times \int_{0}^{1} \operatorname{Ai}^{\prime}\left(z+\xi+\tau\left(g_{N}(\xi)-\xi\right)\right) d \tau \\
& =\frac{\xi^{2}}{\alpha_{N} N^{2 / 3}} L_{N}(\xi) U_{N}(\xi, z) .
\end{aligned}
$$

The second integral in (3.32) is treated analogously. We conclude from (3.31), (3.32) that (3.18) holds where $Q_{1,4}$ is as in (3.22). The proof of Proposition 3.1 is complete.

Now we prove the estimate (3.8) for $\xi, \eta \in I_{N}$. Note that by (3.15) it follows that $g_{N}(\xi), g_{N}(\eta)$ are bounded below by some constant $M_{0}$, and hence in the region $(\xi, \eta) \in I_{N} \times I_{N}$, both variables are bounded below by the constant $L_{0}$. Using in addition (3.16) we conclude that we can always use the exponenial bounds on Ai and its derivatives in (3.9), and hence for any $m \in \mathbb{N}$ and $k=0,1,2$, as $N \rightarrow \infty$

$$
\left|\xi^{m}\left(\frac{d}{d \xi}\right)^{k} \operatorname{Ai}\left(g_{N}(\xi)\right)\right| \leq C(m) e^{-c(m) \xi}, \quad \xi \in I_{N} .
$$

Consider $Q_{1,1}(\xi, \eta)$ first. Recall from (3.7) that, in particular, $\frac{d^{j}}{d z^{j}} R(z)=O\left(N^{-1}\right)$, $j=1,2,3$, uniformly for $|z-1| \leq \delta$. It follows then by (3.19) using (3.13), (3.17), (3.35) that for $j, k=0,1$

$$
\left|\partial_{\xi}^{j} \partial_{\eta}^{k} Q_{1,1}(\xi, \eta)\right| \leq \text { const } \cdot N^{-4 / 3} e^{-c \xi} e^{-c \eta}
$$

uniformly for $\xi, \eta \in I_{N}$. In the same way we find that for $j, k=0,1$ and $l=2,3,4$

$$
\left|\partial_{\xi}^{j} \partial_{\eta}^{k} Q_{1, l}(\xi, \eta)\right| \leq \mathrm{const} \cdot N^{-2 / 3} e^{-c \xi} e^{-c \eta}
$$

uniformly for $\xi, \eta \in I_{N}$. In estimating $Q_{1,4}$, we use the estimate

$$
\left|g_{N}(\xi)-\xi\right| \leq C \delta|\xi|, \quad|\xi| \leq \delta \alpha_{N} N^{2 / 3},
$$

which follows from (3.33), together with the uniform boundedness of $L_{N}(\xi)$ (see (3.10) (4)): for $\delta$ sufficiently small this implies that

$$
\left|U_{N}(\xi, z)\right| \leq C e^{-c z} e^{-c \xi}, \quad \xi \in I_{N}, \quad z \geq 0,
$$

with similar estimates for the $\xi$ - (and $z$-) derivatives. This proves 3.8 for $(\xi, \eta) \in$ $I_{N} \times I_{N}$.

3.2.3. Estimates for $(\xi, \eta) \in I I_{N} \times I I_{N}$. Recall from [ibid., (4.30), (4.31), (6.16)]

$$
S^{(\infty)}(z) \equiv N(z)=\frac{1}{2}\left(\begin{array}{cc}
a(z)+a(z)^{-1} & i\left(a(z)^{-1}-a(z)\right) \\
i\left(a(z)-a(z)^{-1}\right) & a(z)+a(z)^{-1}
\end{array}\right)
$$

where $a(z) \equiv\left(\frac{z-1}{z+1}\right)^{1 / 4} \rightarrow 1$ as $z \rightarrow \infty$.

Proposition 3.2. For $j, k=0,1$ and some $C, c>0$

$$
\left|\partial_{\xi}^{j} \partial_{\eta}^{k}\left(\frac{c_{N}}{\alpha_{N} N^{2 / 3}} K_{N}\left(\xi^{(N)}, \eta^{(N)}\right)\right)\right| \leq C e^{-c N} e^{-c N\left(\xi_{N}-\delta\right)} e^{-c N\left(\eta_{N}-\delta\right)}
$$

uniformly for $\xi, \eta \in I I_{N}$. 
Proof. Note first of all that (3.23) still holds. For $z=1+\xi_{N} \in[1+\delta,+\infty)$ we now have in place of (3.24)

$$
S_{+}(z)=c_{N}^{-N \sigma_{3}} e^{-\frac{N l}{2} \sigma_{3}} Y_{+}\left(c_{N} z+d_{N}\right) e^{-N\left(g_{+}(z)-\frac{l}{2}\right) \sigma_{3}}
$$

where again the constant $l \equiv l_{N}$ is given by [ibid., (5.35)]. Solving for $Y_{+}$and substituting in (3.23) we find for $\xi, \eta \in I I_{N}$

$$
\begin{aligned}
\frac{c_{N}}{\alpha_{N} N^{2 / 3}} K_{N}\left(\xi^{(N)}, \eta^{(N)}\right)= & -e^{-\frac{N}{2}\left(V_{N}\left(1+\xi_{N}\right)-2 g_{+}\left(1+\xi_{N}\right)+l\right)} e^{-\frac{N}{2}\left(V_{N}\left(1+\eta_{N}\right)-2 g_{+}\left(1+\eta_{N}\right)+l\right)} \\
& \times \frac{\left(\begin{array}{ll}
1 & 0
\end{array}\right) \cdot S_{+}^{T}\left(1+\eta_{N}\right) S_{+}^{-T}\left(1+\xi_{N}\right) \cdot\left(\begin{array}{ll}
0 & 1
\end{array}\right)^{T}}{2 \pi i(\xi-\eta)} .
\end{aligned}
$$

In view of [ibid., (5.38)]

$$
-V_{N}\left(1+\xi_{N}\right)+2 g_{+}\left(1+\xi_{N}\right)-l=\Xi_{N,+}\left(1+\xi_{N}\right), \quad \xi \in I I_{N} .
$$

Now by [ibid., (2.14), (5.34)] for some $C_{1}(\delta), C_{2}(\delta)>0$ and $c>0$ for $N$ large enough

$$
\begin{aligned}
\Xi_{N,+}\left(1+\xi_{N}\right) & =-\left(\int_{1}^{1+\delta}+\int_{1+\delta}^{1+\xi_{N}}\right) \sqrt{t^{2}-1} h_{N}(t) d t \\
& \leq-\int_{1}^{1+\delta} \sqrt{t^{2}-1} h_{\min } d t-\int_{1+\delta}^{1+\xi_{N}} c h_{\min } d t \\
& \leq-C_{1}-C_{2}\left(\xi_{N}-\delta\right), \quad \xi \in\left(\delta \alpha_{N} N^{2 / 3},+\infty\right) .
\end{aligned}
$$

By [ibid., (7.46), (7.47)] for $z \geq 1+\delta, S(z)=R(z) S^{(\infty)}(z)$. Using (3.27), which is still valid for $\xi, \eta \in I I_{N}$, we obtain

$$
\begin{aligned}
& S_{+}^{T}\left(1+\eta_{N}\right) S_{+}^{-T}\left(1+\xi_{N}\right)=S_{+}^{(\infty) T}\left(1+\eta_{N}\right) S_{+}^{(\infty)-T}\left(1+\xi_{N}\right) \\
& \quad+\left(\eta_{N}-\xi_{N}\right) S_{+}^{(\infty) T}\left(1+\eta_{N}\right)\left(\int_{0}^{1}\left(R_{+}^{T}\right)^{\prime}\left(1+\xi_{N}+t\left(\eta_{N}-\xi_{N}\right)\right) d t\right) S_{+}^{(\infty)-T}\left(1+\xi_{N}\right) .
\end{aligned}
$$

Substituting

$$
\begin{aligned}
S_{+}^{(\infty) T} & \left(1+\eta_{N}\right)=S_{+}^{(\infty) T}\left(1+\xi_{N}\right) \\
& +\left(\eta_{N}-\xi_{N}\right)\left(\int_{0}^{1}\left(S_{+}^{(\infty) T}\right)^{\prime}\left(1+\xi_{N}+t\left(\eta_{N}-\xi_{N}\right)\right) d t\right.
\end{aligned}
$$

in the first term in the RHS of (3.44) and noting that $\left(\begin{array}{cc}1 & 0\end{array}\right) \cdot I \cdot\left(\begin{array}{ll}0 & 1\end{array}\right)^{T}=0$, we obtain an expression for $\left(\begin{array}{cc}1 & 0\end{array}\right) \cdot S_{+}^{(\infty) T}\left(1+\eta_{N}\right) S_{+}^{(\infty)-T}\left(1+\xi_{N}\right) \cdot\left(\begin{array}{ll}0 & 1\end{array}\right)^{T}$ which is proportional to $(\xi-\eta)$. The exponential bounds (3.40) then follow from (3.43) and the properties of $S^{(\infty)}$ and $R$ (see (3.39) and (3.6), respectively).

Now we prove (3.8) for $\xi, \eta \in I I_{N}$ by showing that both of the two terms on the LHS of (3.8) satisfy the exponential bound. More precisely, let $\xi \in I I_{N}$. Then either $\xi_{N} \geq 2 \delta$ or $\xi_{N} \in[\delta, 2 \delta]$. In the former case

$$
e^{-c N\left(\xi_{N}-\delta\right)}=e^{-c N\left(\left(\xi_{N} / 2\right)-\delta\right)} e^{-\left(c N /\left(2 \alpha_{N} N^{2 / 3}\right)\right) \xi} \leq e^{-\xi}, \quad N \rightarrow \infty,
$$

since $\alpha_{N} \rightarrow(2 m)^{2 / 3} \neq 0$ as $N \rightarrow \infty$. In the latter case

$$
e^{-\xi}=e^{-\alpha_{N} N^{2 / 3} \xi_{N}} \geq e^{-\alpha_{N} N^{2 / 3} 2 \delta} \geq e^{-(c / 2) N}, \quad N \rightarrow \infty
$$


and hence

$$
e^{-c N} e^{-c N\left(\xi_{N}-\delta\right)} \leq e^{-(c / 2) N} e^{-(c / 2) N} \leq e^{-(c / 2) N} e^{-\xi}, \quad \xi_{N} \in[\delta, 2 \delta] .
$$

Combining (3.45) and (3.46) we conclude that Proposition 3.2 implies

$$
\left|\partial_{\xi}^{j} \partial_{\eta}^{k}\left(\frac{c_{N}}{\alpha_{N} N^{2 / 3}} K_{N}\left(\xi^{(N)}, \eta^{(N)}\right)\right)\right| \leq C e^{-c^{\prime} N} e^{-\xi} e^{-\eta}, \quad \xi, \eta \in I I_{N} .
$$

Now we consider $K_{\text {Airy }}(\xi, \eta)$ for $\xi, \eta \in I I_{N}$. It follows from AbSt that

$$
|\operatorname{Ai}(x)|,\left|\operatorname{Ai}^{\prime}(x)\right| \leq C\left(L_{0}\right) e^{-c\left(L_{0}\right)|x|^{3 / 2}}, \quad x \geq L_{0} .
$$

Using the integral representation (1.25) we estimate for $\xi, \eta \in I I_{N}$

$$
\left|K_{\text {Airy }}(\xi, \eta)\right| \leq C \int_{0}^{\infty} e^{-c(z+\xi)^{3 / 2}} e^{-c(z+\eta)^{3 / 2}} d z .
$$

Let $\xi \in I I_{N}$. Then $\xi \geq 1$ for large $N$. It is elementary to verify that

$$
(z+\xi)^{3 / 2} \geq z^{3 / 2}+\xi^{3 / 2}, \quad z \geq 0, \quad \xi \geq 1 .
$$

Next, $\xi^{3 / 2} \geq\left(\delta \alpha_{N}\right)^{3 / 2} N \geq \tilde{c} N, N \rightarrow \infty$ and hence

$$
\xi^{3 / 2}-\xi=\xi^{3 / 2}\left(1-\xi^{-1 / 2}\right) \geq c^{\prime \prime} N, \quad N \rightarrow \infty .
$$

Inserting (3.50), 3.51) and their analogs for $\eta$ in (3.49) we find

$$
\left|K_{\text {Airy }}(\xi, \eta)\right| \leq C e^{-c N} e^{-c \xi} e^{-c \eta}, \quad \xi, \eta \in I I_{N} .
$$

A similar argument using (3.48) also shows that the derivatives of $K_{\text {Airy }}$ satisfy the same bound. Combining (3.47) and (3.52) completes the proof of (3.8) for $\xi, \eta \in I I_{N}$.

3.2.4. The "mixed" neighborhoods of the end point $1:(\xi, \eta) \in\left(I_{N} \times I I_{N}\right) \cup\left(I I_{N} \times\right.$ $\left.I_{N}\right)$. Let us consider the case $(\xi, \eta) \in I_{N} \times I I_{N}$ (the other case is treated analogously). For $K_{\text {Airy }}$, we use the bound in (3.9) for $\xi$,

$$
|\operatorname{Ai}(z+\xi)|,\left|\operatorname{Ai}^{\prime}(z+\xi)\right| \leq C\left(L_{0}\right) e^{-z} e^{-\xi}, \quad z \geq 0, \quad \xi \geq L_{0},
$$

together with the bound (3.48) for $\eta$. Inserting these bounds in (1.25) we obtain for $j, k=0,1$

$$
\left|\partial_{\xi}^{j} \partial_{\eta}^{k} K_{\text {Airy }}(\xi, \eta)\right| \leq C e^{-c N} e^{-c \xi} e^{-c \eta}, \quad(\xi, \eta) \in I_{N} \times I I_{N}
$$

as before. For $K_{N}\left(\xi^{(N)}, \eta^{(N)}\right)$, there are two cases: $\left|\xi_{N}-\eta_{N}\right| \leq \delta / 2$ and $\mid \xi_{N}-$ $\eta_{N} \mid>\delta / 2$. In the first case we can treat both points as lying in a $I_{N} \times I_{N}$ region corresponding to a larger (fixed) value of $\delta$ (more precisely, set $\delta \rightarrow 3 \delta / 2$ ) and hence (3.8) follows by the arguments in Subsection 3.2 .2

It remains to consider the case $(\xi, \eta) \in I_{N} \times I I_{N},\left|\xi_{N}-\eta_{N}\right| \geq \delta / 2$. For such $\xi, \eta$, we have

$$
|\xi-\eta|^{-1} \leq N^{-2 / 3} \alpha_{N}^{-1} 2 \delta^{-1}
$$


The computations that led to (3.26) and (3.42) now imply for $\xi \in I_{N}, \eta \in I I_{N}$

$$
\begin{aligned}
\frac{c_{N}}{\alpha_{N} N^{2 / 3}} & K_{N}\left(\xi^{(N)}, \eta^{(N)}\right)=-\frac{e^{-\pi i / 6} e^{-N \Xi_{N}\left(1+\xi_{N}\right) / 2}}{2 \pi i(\xi-\eta)} \\
& \times\left(\begin{array}{cc}
1 & 0
\end{array}\right) \cdot S^{(\infty) T}\left(1+\eta_{N}\right) R_{+}^{T}\left(1+\eta_{N}\right) R_{+}^{-T}\left(1+\xi_{N}\right) \\
& \times\left(\begin{array}{rr}
1 & -i \\
-1 & -i
\end{array}\right){ }^{-1}\left(\begin{array}{cc}
1 / F_{N}\left(1+\xi_{N}\right) & 0 \\
0 & F_{N}\left(1+\xi_{N}\right)
\end{array}\right) \\
& \times A I_{+}^{-T}\left(f_{N}\left(1+\xi_{N}\right)\right) \cdot\left(\begin{array}{c}
0 \\
1
\end{array}\right),
\end{aligned}
$$

and using the preceding estimates we find for $j, k=0,1$

$$
\left|\partial_{\xi}^{j} \partial_{\eta}^{k} \frac{c_{N}}{\alpha_{N} N^{2 / 3}} K_{N}\left(\xi^{(N)}, \eta^{(N)}\right)\right| \leq C N^{-2 / 3} N^{1 / 6} e^{-c N} e^{-c \xi} e^{-\eta} \leq C e^{-c^{\prime} N} e^{-c \xi} e^{-\eta}
$$

uniformly for $(\xi, \eta) \in I_{N} \times I I_{N},\left|\xi_{N}-\eta_{N}\right|>\delta / 2$.

There is a similar estimate for $(\xi, \eta) \in I I_{N} \times I_{N}$ which, together with 3.53), then proves (3.8) for $(\xi, \eta) \in\left(I_{N} \times I I_{N}\right) \cup\left(I I_{N} \times I_{N}\right)$.

3.3. Estimates on integrals of the CD kernel. For $\xi, \eta \in\left[L_{0},+\infty\right)$, making a change of variables $s=c_{N}\left(1+t_{N}\right)+d_{N}$, and using (3.8) with $j=k=0$, we readily find

$$
\begin{aligned}
& \left|-\int_{\xi^{(N)}}^{\infty} K_{N}\left(s, \eta^{(N)}\right) d s-\left(-\int_{\xi}^{\infty} K_{\text {Airy }}(t, \eta) d t\right)\right| \leq C N^{-2 / 3} e^{-c \xi} e^{-c \eta} \\
& \left|-\int_{\xi^{(N)}}^{\eta^{(N)}} K_{N}\left(s, \eta^{(N)}\right) d s-\left(-\int_{\xi}^{\eta} K_{\text {Airy }}(t, \eta) d t\right)\right| \leq C N^{-2 / 3} e^{-c \min (\xi, \eta)} e^{-c \eta}
\end{aligned}
$$

uniformly for $\xi, \eta \in\left[L_{0},+\infty\right)$.

\section{The CONTRIBUtion OF THE CORRECTION TERM FOR $\beta=1$ AND 4}

4.1. Auxiliary facts concerning integrals of the orthogonal functions $\phi_{j}$. It was shown in [DG (4.14)] that for a fixed $j \in \mathbb{N}$ the following holds as $N \rightarrow \infty$ (see (1.21))

$$
\int_{-\infty}^{+\infty} \phi_{N+j}(y) d y=c_{N}^{1 / 2} N^{-1 / 2}(2 m)^{-1 / 2}\left(1+(-1)^{N+j}+O\left(N^{-1 /(2 m)}\right)\right)
$$

where $2 m=\operatorname{deg} V$. Introduce the following column vectors of size $2 m-1$

$$
\begin{aligned}
& \mathbf{a} \equiv(1,0,1,0, \cdots, 1)^{T}, \quad \mathbf{b} \equiv(0,1,0,1, \cdots, 0)^{T} \\
& \mathbf{e} \equiv \mathbf{a}+\mathbf{b}=(1,1,1, \cdots, 1)^{T} .
\end{aligned}
$$

By (1.47) and (4.1) as (even) $N \rightarrow \infty$

$$
\begin{aligned}
& \epsilon \Phi_{1}(+\infty)=\frac{1}{2} \int_{-\infty}^{+\infty} \Phi_{1}(y) d y=c_{N}^{1 / 2} N^{-1 / 2}(2 m)^{-1 / 2}(\mathbf{b}+o(1)) \\
& \epsilon \Phi_{2}(+\infty)=\frac{1}{2} \int_{-\infty}^{+\infty} \Phi_{2}(y) d y=c_{N}^{1 / 2} N^{-1 / 2}(2 m)^{-1 / 2}(\mathbf{a}+o(1)) .
\end{aligned}
$$

We need also the following result. Recall the notation (1.23), (3.11). 
Proposition 4.1. For any fixed $j \in \mathbb{N}$ there exist $C, c>0$ such that the following holds as $N \rightarrow \infty$,

$$
\left|\phi_{N+j}\left(t^{(N)}\right)-\frac{\alpha_{N}^{1 / 4} N^{1 / 6} 2^{1 / 4}}{c_{N}^{1 / 2}} \operatorname{Ai}(t)\right| \leq C c_{N}^{-1 / 2} N^{-1 / 6} e^{-c t}, \quad t \in I_{N} \cup I I_{N} .
$$

This estimate implies that for a fixed $j \in \mathbb{Z}$ there exist $C, c>0$ such that

$$
\left|\int_{\xi^{(N)}}^{\infty} \phi_{N+j}(s) d s-\frac{c_{N}^{1 / 2}}{N^{1 / 2}} \frac{2^{1 / 4}}{\alpha_{N}^{3 / 4}} \int_{\xi}^{\infty} \operatorname{Ai}(t)\right| \leq C c_{N}^{1 / 2} N^{-5 / 6} e^{-c \xi}, \quad \xi \in I_{N} \cup I I_{N} .
$$

Proof. Assume first that $j=0$. It was shown in DKMVZ2, Thm. 2.2] that (in our notation)

$$
\begin{aligned}
\phi_{N}\left(t^{(N)}\right)=c_{N}^{-1 / 2}[ & \alpha_{N}^{1 / 4} N^{1 / 6} \hat{F}_{N}\left(1+t_{N}\right) \operatorname{Ai}\left(g_{N}(t)\right)\left(1+O\left(N^{-1}\right)\right) \\
& \left.-\alpha_{N}^{-1 / 4} N^{-1 / 6}\left(\hat{F}_{N}\left(1+t_{N}\right)\right)^{-1} \operatorname{Ai}^{\prime}\left(g_{N}(t)\right)\left(1+O\left(N^{-1}\right)\right)\right]
\end{aligned}
$$

where the error terms are uniform for $t \in I_{N}$. Using (3.35) we immediately estimate the second term above by $C c_{N}^{-1 / 2} N^{-1 / 6} e^{-c t}$ uniformly for $t \in I_{N}$. The part of the first term that corresponds to $O\left(N^{-1}\right)$ is estimated similarly by $C c_{N}^{-1 / 2} N^{-5 / 6} e^{-c t}$, $t \in I_{N}$. To estimate the leading part of the first term we write

$$
\begin{aligned}
& \hat{F}_{N}\left(1+t_{N}\right) \operatorname{Ai}\left(g_{N}(t)\right)=\hat{F}_{N}(1) \operatorname{Ai}(t) \\
& \quad+\hat{F}_{N}(1)\left[\operatorname{Ai}\left(g_{N}(t)\right)-\operatorname{Ai}(t)\right]+\operatorname{Ai}\left(g_{N}(t)\right)\left[\hat{F}_{N}\left(1+t_{N}\right)-\hat{F}_{N}(1)\right] .
\end{aligned}
$$

By (3.10) (5) and (3.12), $\hat{F}_{N}(1)=2^{1 / 4}$. By formula (3.34) and (3.38)

$$
\left|\operatorname{Ai}\left(g_{N}(t)\right)-\operatorname{Ai}(t)\right| \leq C N^{-2 / 3} t^{2} e^{-c t} \leq C^{\prime} N^{-2 / 3} e^{-c^{\prime} t}, \quad t \in I_{N} .
$$

Also using (3.35) and (3.17) we obtain

$$
\left|\operatorname{Ai}\left(g_{N}(t)\right)\right| \cdot\left|\hat{F}_{N}\left(1+t_{N}\right)-\hat{F}_{N}(1)\right| \leq C N^{-2 / 3}|t| e^{-c t} \leq C^{\prime} N^{-2 / 3} e^{-c^{\prime} t}, \quad t \in I_{N} .
$$

Combining the above estimates we find that

$$
\left|\hat{F}_{N}\left(1+t_{N}\right) \operatorname{Ai}\left(g_{N}(t)\right)-2^{1 / 4} \operatorname{Ai}(t)\right| \leq C N^{-2 / 3} e^{-c t}, \quad t \in I_{N},
$$

which completes the proof of (4.4) for $j=0$ and $t \in I_{N}$. We now consider (4.4) for $j=0$ and $t \in I I_{N}=\left[\delta \alpha_{N} N^{2 / 3}, \infty\right)$. For such $t$, by (3.9),

$$
|\operatorname{Ai}(t)| \leq C e^{-t} \leq C e^{-c N^{2 / 3}} e^{-t / 2}
$$

and hence $\left|\frac{\alpha_{N}^{1 / 4} N^{1 / 6} 2^{1 / 4}}{c_{N}^{1 / 2}} \operatorname{Ai}(t)\right| \leq C c_{N}^{-1 / 2} N^{-1 / 6} e^{-c t}$. Also from [DG (4.8)], we find $\left|\phi_{N}\left(t^{(N)}\right)\right| \leq C c_{N}^{-1 / 2} e^{-c N} e^{-c t}$. These two estimates for $t \in I I_{N}$, together with the previous estimate for $t \in I_{N}$, yield (4.4) in the case $j=0$ for all $t \in\left[L_{0}, \infty\right)$.

Now fix any $j \in \mathbb{Z}$ and write

$$
\phi_{N+j}\left(c_{N}\left(1+\frac{t}{\alpha_{N} N^{2 / 3}}\right)+d_{N}\right)=\phi_{N+j}\left(c_{N+j}\left(1+\frac{t_{N, j}}{\alpha_{N+j}(N+j)^{2 / 3}}\right)+d_{N+j}\right)
$$


where

$$
\begin{aligned}
t_{N, j}= & t \cdot \frac{c_{N}}{c_{N+j}} \frac{\alpha_{N+j}}{\alpha_{N}} \frac{(N+j)^{2 / 3}}{N^{2 / 3}}+\left(\frac{c_{N}}{c_{N+j}}-1\right) \cdot \alpha_{N+j}(N+j)^{2 / 3} \\
& \quad+\frac{d_{N+j}}{c_{N+j}}\left(\frac{d_{N}}{d_{N+j}}-1\right) \cdot \alpha_{N+j}(N+j)^{2 / 3} \\
= & \left(1+O\left(N^{-1}\right)\right) t+O\left(N^{-1 / 3}\right)
\end{aligned}
$$

by (3.1), (3.2), (3.10) (2). In particular, as $N \rightarrow \infty, t_{N, j} \geq\left(1-\frac{1}{2} \operatorname{sgn} L_{0}\right) L_{0}$. Now the RHS of (4.7) can be written as $\phi_{N^{\prime}}\left(\left(t_{N, j}\right)^{\left(N^{\prime}\right)}\right)$ where $N^{\prime}=N+j$. Applying the estimate (4.4) just derived for $j=0$, with $L_{0}$ replaced by $\left(1-\frac{1}{2} \operatorname{sgn} L_{0}\right) L_{0}$, we obtain

$$
\left|\phi_{N^{\prime}}\left(\left(t_{N, j}\right)^{\left(N^{\prime}\right)}\right)-\frac{\alpha_{N+j}^{1 / 4}(N+j)^{1 / 6} 2^{1 / 4}}{c_{N+j}^{1 / 2}} \operatorname{Ai}\left(t_{N, j}\right)\right| \leq C c_{N+j}^{-1 / 2}(N+j)^{-1 / 6} e^{-c t_{N, j}}
$$

for all $t \geq L_{0}$. Using (4.8), and also (3.1), (3.10) (2), together with the elementary estimate

$$
\left|\operatorname{Ai}\left(t_{N, j}\right)-\operatorname{Ai}(t)\right| \leq C^{\prime} N^{-1 / 3} e^{-c^{\prime} t}
$$

(use (3.9)), we obtain (4.4) from (4.9) for any fixed $j \in \mathbb{Z}$.

Finally (4.5) follows readily by integrating (4.4).

Recall the notation (4.2). Proposition 4.1 implies that for $j=1,2$ one has uniformly for $t, \xi, \eta \geq L_{0}$

$$
\begin{aligned}
& \left|\Phi_{j}\left(t^{(N)}\right)-\frac{\alpha_{N}^{1 / 4} N^{1 / 6} 2^{1 / 4}}{c_{N}^{1 / 2}} \operatorname{Ai}(t) \mathbf{e}\right| \leq C c_{N}^{-1 / 2} N^{-1 / 6} e^{-c t} \\
& \left|\int_{\xi^{(N)}}^{\infty} \Phi_{j}(s) d s-\left(\frac{c_{N}^{1 / 2}}{N^{1 / 2}} \frac{2^{1 / 4}}{\alpha_{N}^{3 / 4}} \int_{\xi}^{\infty} \operatorname{Ai}(t) d t\right) \mathbf{e}\right| \leq C c_{N}^{1 / 2} N^{-5 / 6} e^{-c \xi} \\
& \left|\int_{\xi^{(N)}}^{\eta^{(N)}} \Phi_{j}(s) d s-\left(\frac{c_{N}^{1 / 2}}{N^{1 / 2}} \frac{2^{1 / 4}}{\alpha_{N}^{3 / 4}} \int_{\xi}^{\eta} \operatorname{Ai}(t) d t\right) \mathbf{e}\right| \leq C c_{N}^{1 / 2} N^{-5 / 6} e^{-c \min (\xi, \eta)}
\end{aligned}
$$

4.2. The case $\beta=4$.

4.2.1. The contribution of the correction term to the 12 entry of $K_{N, 4}$. Since $(S D)(x, y)=$ $-\partial_{y} S(x, y)$, the correction term in (1.43) has the form

$$
-\Phi_{2}(x)^{T} \cdot D_{21} \cdot \Phi_{1}(y)-\Phi_{2}(x)^{T} \cdot D_{21} C_{11}^{-1} B_{11} D_{12} \cdot \Phi_{2}(y) .
$$

Set $x=\xi^{(N)}, y=\eta^{(N)}$. Recall $n=2 m-1,2 m=\operatorname{deg} V$. Note that by [DG, (2.13)]

$$
D_{21}=\frac{m \kappa_{2 m}}{2^{2 m-1}} c_{N}^{2 m-1}\left[\left(\begin{array}{ccccc}
\left(\begin{array}{c}
n \\
0
\end{array}\right) & 0 & \left(\begin{array}{c}
n \\
1
\end{array}\right) & \cdots & \left(\begin{array}{c}
n \\
(n-1) / 2
\end{array}\right) \\
0 & 1 & 0 & \cdots & 0 \\
\cdots & & & & \\
0 & 0 & 0 & \cdots & 1
\end{array}\right)+o(1)\right]
$$

and $D_{21}=O\left(c_{N}^{2 m-1}\right)=O\left(N^{1-1 /(2 m)}\right)$ as $N \rightarrow \infty$ (see (3.1) $)$. Also since $C_{11}=$ $I-B_{12} D_{21}$, we see from [ibid., (2.19)] that $C_{11}^{-1} B_{11}$ is skew symmetric, being the lower right corner of the skew symmetric matrix $D_{N}^{-1}$. (Note that $B_{11}$ is the lower right $n \times n$ corner of $\epsilon_{N}$.) Hence $D_{21} C_{11}^{-1} B_{11} D_{12}$ in (4.11) is also skew, and using 
[ibid., (2.13)] and the fact that $C_{11}^{-1}$ is bounded as $N \rightarrow \infty$ [ibid., Thm. 2.4, 2.6], we see that $D_{21} C_{11}^{-1} B_{11} D_{12}=O\left(N^{1-1 /(2 m)}\right)$ as $N \rightarrow \infty$. Recall that the 12 entry in $K_{N, 4}\left(\xi^{(N)}, \eta^{(N)}\right)$ has an overall scaling factor $\left(\frac{c_{N}}{\alpha_{N} N^{2 / 3}}\right)^{2}$. Substituting the leading term in the representation of $\Phi_{j}$ in (4.10) into the first term in (4.11), and using (4.12), we obtain

$$
-\frac{c_{N}^{2}}{\alpha_{N}^{2} N^{4 / 3}} \frac{m \kappa_{2 m}}{2^{2 m-1}} c_{N}^{2 m-1} \frac{\alpha_{N}^{1 / 2} N^{1 / 3} 2^{1 / 2}}{c_{N}}\left(\Sigma_{n}+o(1)\right) \operatorname{Ai}(\xi) \operatorname{Ai}(\eta)
$$

where $o(1)$ is independent of $\xi, \eta$ and $\Sigma_{n}$ denotes the sum of all elements of the first (binomial) matrix on the RHS in (4.12). Using the formula preceding [ibid., (6.7)] one finds

$$
\Sigma_{n}=\frac{1}{2} \frac{m(2 m) !}{(m !)^{2}}
$$

Recall that by (3.10) (2), [ibid., (2.14)] and (3.9),

$$
\begin{aligned}
& \alpha_{N}=2 m^{2 / 3}+o(1), \quad \frac{c_{N}^{2 m}}{N} \frac{m \kappa_{2 m}}{2^{2 m-1}}=\frac{2(m !)^{2}}{(2 m) !}+o(1), \quad N \rightarrow \infty \\
& |\operatorname{Ai}(\xi)| \leq C e^{-\xi}, \quad \xi \geq L_{0} .
\end{aligned}
$$

Inserting these estimates, (4.13) becomes

$$
-\frac{1}{2} \operatorname{Ai}(\xi) \operatorname{Ai}(\eta)+o(1) e^{-\xi} e^{-\eta}
$$

uniformly for $\xi, \eta \geq L_{0}$ and $o(1)$ is independent of $\xi, \eta$. The error that was made by substituting only the leading term in (4.10) in the first term in (4.13), is estimated as follows:

$$
\begin{aligned}
& O\left(c_{N}^{2} N^{-4 / 3}\right) O\left(c_{N}^{2 m-1}\right)\left\{O\left(N^{1 / 6} c_{N}^{-1 / 2}\right) c_{N}^{-1 / 2} N^{-1 / 6}\left(|\operatorname{Ai}(\xi)| e^{-c \eta}+|\operatorname{Ai}(\eta)| e^{-c \xi}\right)\right. \\
& \left.+O\left(N^{-1 / 3} c_{N}^{-1}\right) e^{-c \xi} e^{-c \eta}\right\} \\
& \leq O\left(N^{-1 / 3}\right) e^{-c \xi} e^{-c \eta}
\end{aligned}
$$

uniformly for $\xi, \eta \geq L_{0}$, and independent of the degree $2 m$ of $V$.

Next we substitute the leading terms in the representation of $\Phi_{2}$ in (4.10) in the second term in (4.11). By the skew symmetry of $D_{21} C_{11}^{-1} B_{11} D_{12}$ noted above, the result is precisely zero. The error that is made by such a substitution is estimated in exactly the same way as in 4.15) and is also of order

$$
O\left(N^{-1 / 3}\right) e^{-c \xi} e^{-c \eta}
$$

uniformly for $\xi, \eta \geq L_{0}$. We conclude that the contribution of the correction term to the 12 entry is given by

$$
-\frac{1}{2} \operatorname{Ai}(\xi) \operatorname{Ai}(\eta)+o(1) e^{-c \xi} e^{-c \eta}
$$

uniformly for $\xi, \eta \geq L_{0}$. 
4.2.2. The contribution of the correction term to the 11 and 22 entries of $K_{N, 4}$. We consider the 11 entry of $K_{N, 4}$ (the 22 entry is analyzed in the same way). The correstion term in (1.48) has the form

$$
\begin{aligned}
\Phi_{2}(x)^{T} \cdot D_{21} \cdot\left(-\int_{y}^{\infty} \Phi_{1}(t) d t\right) \\
\quad+\Phi_{2}(x)^{T} \cdot D_{21} C_{11}^{-1} B_{11} D_{12} \cdot\left(-\int_{y}^{\infty} \Phi_{2}(t) d t\right) .
\end{aligned}
$$

We set $x=\xi^{(N)}, y=\eta^{(N)}$ in (4.18). The 11 (and 22) entry in $K_{N, 4}\left(\xi^{(N)}, \eta^{(N)}\right.$ ) has an overall scaling factor $\frac{c_{N}}{\alpha_{N} N^{2 / 3}}$. Hence, substituting the leading terms in the representation of $\Phi_{2}, \int \Phi_{1}$ in (4.10) into the first term in (4.18) and using (4.12), we obtain

$$
\frac{c_{N}}{\alpha_{N} N^{2 / 3}} \frac{m \kappa_{2 m}}{2^{2 m-1}} c_{N}^{2 m-1} \frac{\alpha_{N}^{1 / 4} N^{1 / 6} 2^{1 / 4}}{c_{N}^{1 / 2}} \frac{c_{N}^{1 / 2} 2^{1 / 4}}{\alpha_{N}^{3 / 4} N^{1 / 2}}\left(\Sigma_{n}+o(1)\right) \operatorname{Ai}(\xi)\left(-\int_{\eta}^{\infty} \operatorname{Ai}(t) d t\right)
$$

where $o(1)$ is independent of $\xi, \eta$. Computing the factor and using (4.14) as above we see that (4.19) becomes

$$
-\frac{1}{2} \operatorname{Ai}(\xi) \int_{\eta}^{\infty} \operatorname{Ai}(t) d t+o(1) e^{-\xi} e^{-\eta}
$$

uniformly for $\xi, \eta \geq L_{0}$ and $o(1)$ is independent of $\xi, \eta$. The error that was made by substituting only the leading terms for $\Phi_{2}, \int \Phi_{1}$ in (4.10) into the first term in (4.18), is estimated as follows:

$$
\begin{aligned}
O\left(c_{N} N^{-2 / 3}\right) O\left(c_{N}^{2 m-1}\right)( & \left.\frac{N^{1 / 6}}{c_{N}^{1 / 2}} \frac{c_{N}^{1 / 2}}{N^{5 / 6}}+\frac{1}{N^{1 / 6} c_{N}^{1 / 2}} \frac{c_{N}^{1 / 2}}{N^{1 / 2}}+\frac{c_{N}^{1 / 2}}{N^{5 / 6}} \frac{1}{N^{1 / 6} c_{N}^{1 / 2}}\right) e^{-c \xi} e^{-c \eta} \\
& =O\left(N^{1 / 3}\right)\left(N^{-2 / 3}+N^{-2 / 3}+N^{-1}\right) e^{-c \xi} e^{-c \eta} \\
& =O\left(N^{-1 / 3}\right) e^{-c \xi} e^{-c \eta}
\end{aligned}
$$

uniformly for $\xi, \eta \geq L_{0}$, and again independent of the degree $2 m$ of $V$.

Next we substitute the leading terms in the representation of $\Phi_{2}, \int \Phi_{2}$ into 4.10) in the second term in (4.18). Again by skew symmetry, the result is precisely zero. The error that is made by such a substitution is estimated in exactly the same way as in (4.20) and also has order

$$
O\left(N^{-1 / 3}\right) e^{-c \xi} e^{-c \eta}
$$

uniformly for $\xi, \eta \geq L_{0}$. We conclude that the contribution of the correction term to the 11 entry is given by

$$
-\frac{1}{2} \operatorname{Ai}(\xi) \int_{\eta}^{\infty} \operatorname{Ai}(t) d t+o(1) e^{-c \xi} e^{-c \eta}
$$

uniformly for $\xi, \eta \geq L_{0}$ (for the 22 entry $\xi$ and $\eta$ should be interchanged). 
4.2.3. The contribution of the correction term to the 21 entry of $K_{N, 4}$. By (1.48), (1.50) the correction term in the 21 entry of $K_{N, 4}$ is given by

$$
\begin{aligned}
\int_{x}^{\infty} \Phi_{2}^{T}(s) & d s \cdot D_{21} \cdot \int_{y}^{\infty} \Phi_{1}(t) d t \\
& +\int_{x}^{\infty} \Phi_{2}^{T}(s) d s \cdot D_{21} C_{11}^{-1} B_{11} D_{12} \cdot \int_{y}^{\infty} \Phi_{2}(t) d t .
\end{aligned}
$$

Again we replace $x=\xi^{(N)}, y=\eta^{(N)}$. Recall that the 21 entry in $K_{N, 4}\left(\xi^{(N)}, \eta^{(N)}\right)$ has no overall scaling factor. Substituting the leading terms in the representation of $\int \Phi_{j}, j=1,2$, in (4.10) into the first term in 4.23) in the same way as before, we obtain

$$
\begin{aligned}
\frac{m \kappa_{2 m}}{2^{2 m-1}} c_{N}^{2 m-1} & \frac{c_{N} 2^{1 / 2}}{\alpha_{N}^{3 / 2} N}\left(\Sigma_{n}+o(1)\right) \int_{\xi}^{\infty} \operatorname{Ai}(s) d s \int_{\eta}^{\infty} \operatorname{Ai}(t) d t \\
& =\frac{1}{2} \int_{\xi}^{\infty} \operatorname{Ai}(s) d s \int_{\eta}^{\infty} \operatorname{Ai}(t) d t+o(1) e^{-\xi} e^{-\eta}
\end{aligned}
$$

uniformly for $\xi, \eta \geq L_{0}$ and $o(1)$ is independent of $\xi, \eta$. The error just made is estimated as follows:

$$
\begin{aligned}
O\left(c_{N}^{2 m-1}\right)\left(2 \frac{c_{N}^{1 / 2}}{N^{1 / 2}} \frac{c_{N}^{1 / 2}}{N^{5 / 6}}+\frac{c_{N}}{N^{5 / 3}}\right) e^{-c \xi} e^{-c \eta} \\
=O\left(N^{1 / 3}\right)\left(N^{-4 / 3}+N^{-5 / 3}\right) e^{-c \xi} e^{-c \eta} \\
=O\left(N^{-1 / 3}\right) e^{-c \xi} e^{-c \eta}
\end{aligned}
$$

uniformly for $\xi, \eta \geq L_{0}$, here all order factors are independent of $\xi, \eta$. (Here we have used $\left|\int_{\xi}^{\infty} \operatorname{Ai}(t) d t\right| \leq C e^{-\xi}$ uniformly for $\xi \geq L_{0}$.)

Finally, we substitute the leading terms in the representation of $\int \Phi_{j}, j=1,2$, in (4.10) into the second term in 4.23). By the skew symmetry the result is again precislely zero. The error that is made by such a substitution is estimated in exactly the same way as in (4.24) and is also of order

$$
O\left(N^{-1 / 3}\right) e^{-c \xi} e^{-c \eta}
$$

uniformly for $\xi, \eta \geq L_{0}$. We conclude that the contribution of the correction term to the 21 entry is given by

$$
\frac{1}{2} \int_{\xi}^{\infty} \operatorname{Ai}(s) d s \int_{\eta}^{\infty} \operatorname{Ai}(t) d t+o(1) e^{-c \xi} e^{-c \eta}
$$

uniformly for $\xi, \eta \geq L_{0}$.

4.3. The case $\beta=1$. As we will see, this case is more involved than the case $\beta=4$. Consider the $2 n \times 2 n(n=2 m-1,2 m=\operatorname{deg} V)$ matrix $\left(A C\left(I_{2 n}-B A C\right)^{-1}\right)^{T}$ in the $\beta=1$ correction term in (1.42) as a two by two block matrix with blocks of size $n \times n$. Denote the upper left and the upper right blocks by $G_{11}$ and $G_{12}$, respectively. With this notation the correction term has the form

$$
-\Phi_{1}(x)^{T} \cdot G_{11} \cdot \epsilon \Phi_{1}(y)-\Phi_{1}(x)^{T} \cdot G_{12} \cdot \epsilon \Phi_{2}(y) .
$$

As in [DG] let $R \equiv R_{n}$ denote the $n \times n$ matrix with all entries zero apart from ones on the anti-diagonal (thus $R_{i, j}=1$ if $j=n-i+1,1 \leq i \leq n$, and $R_{i, j}=0$ 
otherwise). Note that $R^{2}=I_{n}$. Define

$$
\tilde{G}_{11} \equiv-R D_{21} C_{11}^{-1} B_{11} D_{12} R \text {. }
$$

Note from Subsection 4.2.1 that $D_{21} C_{11}^{-1} B_{11} D_{12}$ is skew and of order $O\left(N^{1-1 /(2 m)}\right)$ as $N \rightarrow \infty$. Hence $\tilde{G}_{11}$ is also skew and has the same order as $N \rightarrow \infty$. We need the following result.

Proposition 4.2. As (even) $N \rightarrow \infty$ we have $G_{11}, G_{12}=O\left(N^{1-1 /(2 m)}\right)$, more precisely

$$
G_{11}=\tilde{G}_{11}+o\left(N^{1-1 /(2 m)}\right), \quad N \rightarrow \infty
$$

and also

$$
G_{12}=D_{12}+o\left(N^{1-1 /(2 m)}\right), \quad N \rightarrow \infty .
$$

Proof. It was shown in [DG. Theorem 2.3] that, as $N \rightarrow \infty$,

$$
\begin{aligned}
(B A)_{22} & =-R(B A)_{11} R+o(1) \\
B A C & =\left(\begin{array}{cc}
0 & 0 \\
(B A)_{21}+o(1) & (B A)_{22}+o(1)
\end{array}\right) .
\end{aligned}
$$

Denote

(4.32)

$T \equiv I_{n}-(B A C)_{22}=I_{n}-(B A)_{22}+o(1)=I_{n}+R(B A)_{11} R+o(1)=R C_{11} R+o(1)$.

It was shown in DG. Theorem 2.6] that, as $N \rightarrow \infty, T$ approaches a constant nondegenerate matrix. Thus

$$
\left(I_{2 n}-B A C\right)^{-1}=\left(\begin{array}{cc}
I_{n} & 0 \\
T^{-1}\left((B A)_{21}+o(1)\right) & T^{-1}
\end{array}\right)
$$

and simple algebra using (1.39), (1.40) now shows that in the product $A C\left(I_{2 n}-\right.$ $B A C)^{-1}=\left(\begin{array}{cc}G_{11}^{T} & * \\ G_{12}^{T} & *\end{array}\right)$ we have by (4.31)

$$
\begin{aligned}
& G_{11}^{T}=A_{12}\left[(B A)_{21}+(B A)_{22} T^{-1}\left((B A)_{21}+o(1)\right)\right] \\
& G_{12}^{T}=A_{21}\left[I_{n}+(B A)_{11}+(B A)_{12} T^{-1}\left((B A)_{21}+o(1)\right)\right] .
\end{aligned}
$$

Using (4.32), this implies

$$
\begin{aligned}
N^{-1+1 /(2 m)} G_{11}^{T} & =N^{-1+1 /(2 m)} A_{12}\left[I_{n}+(B A)_{22} T^{-1}\right](B A)_{21}+o(1) \\
& =N^{-1+1 /(2 m)} A_{12}\left[T+(B A)_{22}\right] T^{-1}(B A)_{21}+o(1) \\
& =N^{-1+1 /(2 m)} A_{12} T^{-1}(B A)_{21}+o(1) .
\end{aligned}
$$

Now from

$$
B A=\left(\begin{array}{ll}
B_{12} A_{21} & B_{11} A_{12} \\
B_{22} A_{21} & B_{21} A_{12}
\end{array}\right)
$$


and (4.34), 4.32) we obtain

$$
\begin{aligned}
N^{-1+1 /(2 m)} G_{11}^{T} & =N^{-1+1 /(2 m)} A_{12}\left(R R+R(B A)_{11} R\right)^{-1} B_{22} A_{21}+o(1) \\
& =N^{-1+1 /(2 m)} A_{12} R\left(I_{n}+(B A)_{11}\right)^{-1} R B_{22} A_{21}+o(1) \\
& =N^{-1+1 /(2 m)} A_{12} R C_{11}^{-1} R B_{22} R R A_{21}+o(1) .
\end{aligned}
$$

Using the asymptotic relations

$$
\begin{aligned}
N^{-1+1 /(2 m)} R A_{12} R & =N^{-1+1 /(2 m)} A_{21}+o(1) \\
N^{1-1 /(2 m)} R B_{22} R & =-N^{1-1 /(2 m)} B_{11}+o(1)
\end{aligned}
$$

from DG, Subsec. 5.2] we see that

$$
\begin{aligned}
N^{-1+1 /(2 m)} R G_{11}^{T} R & =N^{-1+1 /(2 m)}\left(R A_{12} R\right) C_{11}^{-1}\left(R B_{22} R\right)\left(R A_{21} R\right)+o(1) \\
& =-N^{-1+1 /(2 m)} A_{21} C_{11}^{-1} B_{11} A_{12}+o(1) \\
& =N^{-1+1 /(2 m)} D_{21} C_{11}^{-1} B_{11} D_{12}+o(1) .
\end{aligned}
$$

As noted above, the matrix $D_{21} C_{11}^{-1} B_{11} D_{12}$ is skew symmetric and hence

$$
N^{-1+1 /(2 m)} G_{11}=-N^{-1+1 /(2 m)} R D_{21} C_{11}^{-1} B_{11} D_{12} R+o(1)
$$

which proves 4.29).

Now let us prove 4.30). From 4.33) we derive

$$
N^{-1+1 /(2 m)} G_{12}^{T}=N^{-1+1 /(2 m)} A_{21}\left[I_{n}+(B A)_{11}+(B A)_{12} T^{-1}(B A)_{21}\right]+o(1)
$$

and hence, because $A_{21}^{T}=A_{12}=D_{12}$, we note that we just have to prove

$$
N^{-1+1 /(2 m)} A_{21}\left[(B A)_{11}+(B A)_{12} T^{-1}(B A)_{21}\right]=o(1) .
$$

Since $N^{-1+1 /(2 m)} A_{21}=O(1)$, it is sufficient to prove

$$
(B A)_{11}+(B A)_{12} T^{-1}(B A)_{21}=o(1) .
$$

By (4.35) the LHS is $B_{12} A_{21}+B_{11} A_{12} T^{-1} B_{22} A_{21}$ and so we see that it is sufficient to show that

$$
B_{12}+B_{11} A_{12} T^{-1} B_{22}=o\left(N^{-1+1 /(2 m)}\right) .
$$

Using (4.32) this reduces to showing that

$$
B_{12}+B_{11} A_{12} R C_{11}^{-1} R B_{22}=o\left(N^{-1+1 /(2 m)}\right)
$$

or

$$
R B_{12} R+\left(R B_{11} R\right)\left(R A_{12} R\right) C_{11}^{-1}\left(R B_{22} R\right)=o\left(N^{-1+1 /(2 m)}\right) .
$$

Using $N^{-1+1 /(2 m)} R B_{12} R=-N^{-1+1 /(2 m)} B_{21}+o(1)$ which follows as in (4.36), we are reduced to proving finally

$$
-B_{21}+B_{22} A_{21} C_{11}^{-1} B_{11}=o\left(N^{-1+1 /(2 m)}\right)
$$

But

$$
-B_{21}+B_{22} A_{21} C_{11}^{-1} B_{11}=0
$$

by (taking the transposes of) DG, (5.12)]. The proof of Proposition 4.2 is complete. 
Remark 4.1. The second relation in (4.31) was sharpened recently by Kriecherbauer and Vanlessen [KV] who showed that the $o(1)$ terms are in fact identically zero. One might hope that this improved result could be used to strengthen the estimates in (4.29), (4.30). This is indeed the case for (4.30): one can show that $G_{12}=D_{12}$ identically. However we have not been able to use $[\mathrm{KV}]$ to improve the estimate in (4.29).

4.3.1. The contribution of the correction term to the 12 entry of $K_{N, 1}$. In view of (4.27), since $(S D)(x, y)=-\partial_{y} S(x, y)$, the correction term has the form

$$
\Phi_{1}(x)^{T} \cdot G_{11} \cdot \Phi_{1}(y)+\Phi_{1}(x)^{T} \cdot G_{12} \cdot \Phi_{2}(y) .
$$

Again set $x=\xi^{(N)}, y=\eta^{(N)}$. Using Proposition 4.2 and proceeding in the same way as in Subsection 4.2.1 we find that as $N \rightarrow \infty$, the term (4.38), multiplied as before by $\left(\frac{c_{N}}{\alpha_{N} N^{2 / 3}}\right)^{2}$, becomes

$$
-\frac{1}{2} \operatorname{Ai}(\xi) \operatorname{Ai}(\eta)+o(1) e^{-c \xi} e^{-c \eta}
$$

uniformly for $\xi, \eta \geq L_{0}$. Note that the sum of all elements of (the binomial matrix in the limiting form of) $D_{12}$ is, up to a sign, the same as for $D_{21}$.

Remark: Note also that the only new element in the above proof as compared with the case $\beta=4$ in Subsection 4.2 .1 is that the matrix $G_{11}$ is only asymptotically (and not identically) skew symmetric. This leads to the estimate $o(1) e^{-c \xi} e^{-c \eta}$ in place of (4.16).

4.3.2. The contribution of the correction term to the 11 and 22 entries of $K_{N, 1}$. We consider the 11 entry of $K_{N, 1}$ (the 22 entry is considered in the same way). Using (1.47), (4.27) we rewrite the correction term as

$$
\begin{gathered}
-\Phi_{1}(x)^{T} \cdot G_{11} \cdot\left(-\int_{y}^{\infty} \Phi_{1}(t) d t\right)-\Phi_{1}(x)^{T} \cdot G_{12} \cdot\left(-\int_{y}^{\infty} \Phi_{2}(t) d t\right) \\
-\Phi_{1}(x)^{T} \cdot G_{11} \cdot \epsilon \Phi_{1}(+\infty)-\Phi_{1}(x)^{T} \cdot G_{12} \cdot \epsilon \Phi_{2}(+\infty) .
\end{gathered}
$$

Again set $x=\xi^{(N)}, y=\eta^{(N)}$. The first two terms can be treated in the same way as in Subsections 4.2.2 and 4.3.1. More precisely we find that the first two terms in (4.40), multiplied by $\frac{c_{N}}{\alpha_{N} N^{2 / 3}}$, become, as $N \rightarrow \infty$

$$
-\frac{1}{2} \operatorname{Ai}(\xi) \int_{\eta}^{\infty} \operatorname{Ai}(t) d t+o(1) e^{-c \xi} e^{-c \eta}
$$

uniformly for $\xi, \eta \geq L_{0}$. Now consider the (scaled) sum of the last two terms in (4.40)

$$
-\frac{c_{N}}{\alpha_{N} N^{2 / 3}}\left(\Phi_{1}\left(\xi^{(N)}\right)^{T} \cdot G_{11} \cdot \epsilon \Phi_{1}(+\infty)+\Phi_{1}\left(\xi^{(N)}\right)^{T} \cdot G_{12} \cdot \epsilon \Phi_{2}(+\infty)\right) .
$$

By (4.3), 4.10), Proposition 4.2 this becomes as $N \rightarrow \infty$

$$
\begin{aligned}
-\frac{c_{N}}{\alpha_{N} N^{2 / 3}} & \frac{\alpha_{N}^{1 / 4} N^{1 / 6} 2^{1 / 4}}{c_{N}^{1 / 2}} \frac{c_{N}^{1 / 2}}{(2 m)^{1 / 2} N^{1 / 2}} \\
& \times\left\{\mathbf{e}^{T} \cdot G_{11} \cdot(\mathbf{b}+o(1))+\mathbf{e}^{T} \cdot G_{12} \cdot(\mathbf{a}+o(1))\right\} \mathrm{Ai}(\xi)+o(1) e^{-c \xi}
\end{aligned}
$$


Setting $\mathbf{a}=\mathbf{e}-\mathbf{b}$, we find that (4.43) reduces to (4.44)

$$
\frac{1}{2} \operatorname{Ai}(\xi)+o(1) e^{-c \xi}+\left(\mathbf{e}^{T} \cdot G_{11} \cdot \mathbf{b}-\mathbf{e}^{T} \cdot G_{12} \cdot \mathbf{b}\right) \cdot \operatorname{Ai}(\xi) \cdot O\left(N^{-1+1 /(2 m)}\right) .
$$

So if we could prove

$$
\mathbf{e}^{T} \cdot G_{11} \cdot \mathbf{b}-\mathbf{e}^{T} \cdot G_{12} \cdot \mathbf{b}=o\left(N^{1-1 /(2 m)}\right), \quad N \rightarrow \infty,
$$

then we would find that (4.44) equals

$$
\frac{1}{2} \operatorname{Ai}(\xi)+o(1) e^{-c \xi}
$$

uniformly for $\xi \geq L_{0}$.

We prove (4.45). We will, perhaps surprisingly, use a property of the $\beta=4$ correlation kernel $S_{N / 2,4}$ : it is not clear how to prove 4.45) directly using the asymptotic properties of $\left(D \phi_{N+j}, \phi_{N+k}\right)$ and $\left(\epsilon \phi_{N+j}, \phi_{N+k}\right)$ given in DG. More precisely, (4.45) follows from (4.29), (4.30) and the relation

$$
\mathbf{b}+C_{11}^{-1} B_{11} D_{12} \cdot \mathbf{a}=o(1), \quad N \rightarrow \infty,
$$

which is proved by dividing (1.46) by $\left(\frac{c_{N}}{2 m N}\right)^{1 / 2}$ and using (4.3) as $N \rightarrow \infty$. Multiplying (4.47) from the left by $\mathbf{e}^{T} \cdot R D_{21}$ and noting $\mathbf{b}=R \cdot \mathbf{b}, \mathbf{a}=R \cdot \mathbf{a}$, we find

$$
\mathbf{e}^{T} \cdot R D_{21} R \cdot \mathbf{b}+\mathbf{e}^{T} \cdot R D_{21} C_{11}^{-1} B_{11} D_{12} R \cdot(\mathbf{e}-\mathbf{b})=o\left(N^{1-1 /(2 m)}\right) .
$$

But the second matrix is skew symmetric (see (4.28) et seq.). By (4.29) the above relation becomes

$$
\mathbf{e}^{T} \cdot R D_{21} R \cdot \mathbf{b}+\mathbf{e}^{T} \cdot G_{11} \cdot \mathbf{b}=o\left(N^{1-1 /(2 m)}\right) .
$$

But from 4.36

$$
R D_{21} R=-D_{12}+o\left(N^{1-1 /(2 m)}\right)
$$

and hence (4.48), (4.30) imply 4.45).

Collecting the estimates (4.41), (4.46) we see that since $\int_{-\infty}^{\infty} \operatorname{Ai}(t) d t=1$, the correction term in the 11 entry has the form

$$
\begin{aligned}
\frac{1}{2} \operatorname{Ai}(\xi) & \left(1-\int_{\eta}^{\infty} \operatorname{Ai}(t) d t\right)+o(1) e^{-c \xi} \\
& =\frac{1}{2} \operatorname{Ai}(\xi) \int_{-\infty}^{\eta} \operatorname{Ai}(t) d t+o(1) e^{-c \xi}
\end{aligned}
$$

uniformly for $\xi, \eta \geq L_{0}$. The correction term in the 22 entry has the same asymptotic form with $\xi$ and $\eta$ interchanged.

4.3.3. The contribution of the correction term to the 21 entry of $K_{N, 1}$. By (1.49), (4.27) the correction term in this case has the form

$$
\left(\int_{x}^{y} \Phi_{1}(t)^{T} d t\right) \cdot G_{11} \cdot \epsilon \Phi_{1}(y)+\left(\int_{x}^{y} \Phi_{1}(t)^{T} d t\right) \cdot G_{12} \cdot \epsilon \Phi_{2}(y)
$$


which equals

$$
\begin{aligned}
& \left(\int_{x}^{y} \Phi_{1}(t)^{T} d t\right) \cdot G_{11} \cdot\left(-\int_{y}^{\infty} \Phi_{1}(t) d t\right) \\
& \quad+\left(\int_{x}^{y} \Phi_{1}(t)^{T} d t\right) \cdot G_{12} \cdot\left(-\int_{y}^{\infty} \Phi_{2}(t) d t\right) \\
& \quad+\left(\int_{x}^{y} \Phi_{1}(t)^{T} d t\right) \cdot G_{11} \cdot \epsilon \Phi_{1}(+\infty)+\left(\int_{x}^{y} \Phi_{1}(t)^{T} d t\right) \cdot G_{12} \cdot \epsilon \Phi_{2}(+\infty)
\end{aligned}
$$

by (1.47). Again set $x=\xi^{(N)}, y=\eta^{(N)}$. A calculation very similar to the one in Subsection 4.3.2 using the last estimate in (4.10) in place of the first, leads to the following asymptotic form for the 21 correction as $N \rightarrow \infty$

$$
-\frac{1}{2} \int_{\xi}^{\eta} \operatorname{Ai}(s) d s+\frac{1}{2}\left(\int_{\xi}^{\eta} \operatorname{Ai}(s) d s\right)\left(\int_{\eta}^{\infty} \operatorname{Ai}(t) d t\right)+o(1) e^{-c \min (\xi, \eta)}
$$

uniformly for $\xi, \eta \geq L_{0}$. (Recall that there is no overall scaling factor for the 21 entry.) This completes the analysis of the contribution of the correction term to the 21 entry of $K_{N, 1}$.

\section{REFERENCES}

[AbSt] M. Abramowitz and I. A. Stegun, Handbook of Mathematical Functions, with Formulas, Graphs and Mathematical Tables. Dover, New York, 1966.

[AFNvM] M. Adler, P. J. Forrester, T. Nagao and P. van Moerbeke, Classical skew orthogonal polynomials and random matrices. J. Statist. Phys. 99 (2000), 141-170.

[AvM] M. Adler and P. van Moerbeke, Toda versus Pfaff lattice and related polynomials. Duke Math. J. 112 (2002), 1-58.

[Be] C. W. J. Beenakker, Universality for Brézin and Zee's spectral correlator. Nuclear Phys. B 422 (1994), 515-520.

[BI] P. Bleher and A. Its, Semiclassical asymptotics of orthogonal polynomials, Riemann-Hilbert problem, and universality in the matrix model. Ann. of Math. (2) 150 (1999), 185-266.

$[\mathrm{BoBr}] \mathrm{M}$. J. Bowick and E. Brézin, Universal scaling of the tail of the density of eigenvalues in random matrix models. Phys. Lett. B 268 (1991), 21-28.

[BrZ] E. Brézin and A. Zee, Universality of the correlations between eigenvalues of large random matrices. Nuclear Phys. B 402 (1993), 613-627.

$[\mathrm{CKu}] \mathrm{T}$. Claeys and A. B. J. Kuijlaars, Universality of the double scaling limit in random matrix models, preprint, 2005. www.arxiv.org/abs/math-ph/0501074

[DG] P. Deift and D. Gioev, Universality in Random Matrix Theory for for orthogonal and symplectic ensembles, submitted, 2004. www.arxiv.org/abs/math-ph/0411075

[DGKV] P. Deift, D. Gioev, T. Kriecherbauer and M. Vanlessen, Universality for orthogonal and symplectic ensembles of random matrices with generalized Laguerre type weights, in preparation, 2005.

[DKMVZ1] P. Deift, T. Kriecherbauer, K. T.-R. McLaughlin, S. Venakides and X. Zhou, Uniform asymptotics for polynomials orthogonal with respect to varying exponential weights and applications to universality questions in random matrix theory. Comm. Pure Appl. Math. 52 (1999), 1335-1425.

[DKMVZ2] P. Deift, T. Kriecherbauer, K. T.-R. McLaughlin, S. Venakides and X. Zhou, Strong asymptotics of orthogonal polynomials with respect to exponential weights. Comm. Pure Appl. Math. 52 (1999), 1491-1552.

[DVZ] P. Deift, S. Venakides and X. Zhou, New results in small dispersion KdV by an extension of the steepest descent method for Riemann-Hilbert problems. Int. Math. Res. Not. 1997 (1997), 286-299.

[DZ] P. Deift and X. Zhou, A steepest descent method for oscillatory Riemann-Hilbert problems. Asymptotics for the MKdV equation. Ann. of Math. (2) 137 (1993), 295-368.

[Dy] F. J. Dyson, A note on correlations between eigenvalues of a random matrix. Comm. Math. Phys. 19 (1970), 235-250. 
[FoIKi] A. S. Fokas, A. R. Its and A.V. Kitaev, Discrete Painleve equations and their appearance in quantum gravity. Comm. Math. Phys. 142 (1991), 313-344.

[F] P. J. Forrester, The spectrum edge of random matrix ensembles. Nuclear Phys. B 402 (1993), 709-728.

[FNH] P. J. Forrester, T. Nagao and G. Honner, Correlations for the orthogonal-unitary and symplectic-unitary transitions at the hard and soft edges. Nuclear Phys. B 553 (1999), 601-643.

[HWe] G. Hackenbroich and H. A. Weidenmüller, Universality of Random-Matrix results for nonGaussian ensembles. Phys. Rev. Lett. 74 (1995), 4118-4121.

$[\mathrm{KaFr}]$ E. Kanzieper and V. Freilikher, Universality in invariant random-matrix models: Existence near the soft edge. Phys. Rev. E 55 (1997), 3712-3715.

[KV] T. Kriecherbauer and M. Vanlessen, private communication, 2004.

[MaM] G. Mahoux and M. L. Mehta, A method of integration over matrix variables. IV. J. Physique I France 1 (1991), 1093-1108.

[M1] M. L. Mehta, Random Matrices, 2nd Ed., Academic Press, San Diego, 1991.

[M2] M. L. Mehta, A note on correlations between eigenvalues of a random matrix. Comm. Math. Phys. 20 (1971), 245-250.

[MhSa] H. N. Mhaskar and E. B. Saff, Extremal problems for polynomials with exponential weights. Trans. Amer. Math. Soc. 285 (1984), 203-234.

[Mo] G. Moore, Matrix models of $2 D$ quantum gravity and isomonodromy deformations. Progr. Theor. Phys. Suppl. 102 (1990), 255-285.

[NW] T. Nagao and M. Wadati, Correlation functions of random matrix ensembles related to classical orthogonal polynomials, I-III. J. Phys. Soc. Japan 60 (1991), 3298-3322, ibid. 61 (1992), 78-88 and 1910-1918.

[PS] L. Pastur and M. Shcherbina, Universality of the local eigenvalue statistics for a class of unitary invariant random matrix ensembles. J. Statist. Phys. 86 (1997), 109-147.

[Ra] E. A. Rakhmanov, Asymptotic properties of orthogonal polynomials on the real axis. Mat. Sb. (N.S.) 119(161) (1982), 163-203, 303. (Russian). English transl. in: Math. Sb. (N.S.) 47 (1984), 155-193.

[ReSi] M. Reed and B. Simon, Methods of modern mathematical physics, IV, Academic Press, New York-London, 1978.

[SaTo] E. B. Saff and V. Totik, Logarithmic Potentials with External Fields, Grundlehren der Mathematischen Wissenschaften, 316. Springer-Verlag, Berlin, 1997.

[SeVe] M. K. Sener and J. J. M. Verbaarschot, Universality in chiral random matrix theory at $\beta=1$ and $\beta=4$. Phys. Rev. Lett. 81 (1998), 248-251.

[Si] B. Simon, Trace Ideals and Their Applications, London Mathematical Society Lecture Notes Series, 35. Cambridge University Press, Cambridge-New York, 1979.

[So] A. Soshnikov, Universality at the edge of the spectrum in Wigner random matrices. Comm. Math. Phys. 207 (1999), 697-733.

[St1] A. Stojanovic, Des problèmes asymptotiques dans la théorie spectrale des matrices aléatoires. Ph.D. Thesis, Université Paris 7 Denis Diderot, 2003.

[St2] A. Stojanovic, Universality in orthogonal and symplectic invariant matrix models with quartic potential. Math. Phys. Anal. Geom. 3 (2000), 339-373 (2001). Errata: ibid., in press, 2004

[St3] A. Stojanovic, Universalité pour des modèles matriciels à symétrie orthogonale ou symplectique et à potentiel quartique. Preprint 02-07-098, revised version of Preprint 00-01-06. www.physik.uni-bielefeld.de/bibos/preprints

[Sz] G. Szegö, Orthogonal Polynomials, Amer. Math. Soc. Colloq. Publ., v. 23. Amer. Math. Soc., New York, 1939.

[TW1] C. A. Tracy and H. Widom, Fredholm determinants, differential equations and matrix models. Comm. Math. Phys. 163 (1994), 33-72.

[TW2] C. A. Tracy and H. Widom, Correlation functions, cluster functions, and spacing distributions for random matrices. J. Statist. Phys. 92 (1998), 809-835.

[TW3] C. A. Tracy and H. Widom, Matrix kernels for the Gaussian orthogonal and symplectic ensembles, 2004. www.arxiv.org/abs/math-ph/0405035

[TW4] C. A. Tracy and H. Widom, Level-spacing distributions and the Airy kernel. Comm. Math. Phys. 159 (1994), 151-174, announcement in: Phys. Lett. B 305 (1993), 115-118. 
[TW5] C. A. Tracy and H. Widom, On orthogonal and symplectic matrix ensembles. Comm. Math. Phys. 177 (1996), 727-754.

[TW6] C. A. Tracy and H. Widom, Distribution functions for largest eigenvalues and their applications. Proceedings of the International Congress of Mathematicians, Vol. I (Beijing, 2002), 587-596, Higher Ed. Press, Beijing, 2002.

[V] M. Vanlessen, Strong asymptotics of Laguerre-type orthogonal polynomials and applications in random matrix theory, preprint, 2005. www.arxiv.org/abs/math.CA/0504604

[Ve] J. Verbaarschot, Topics in Random Matrix Theory, lecture notes. tonic.physics. sunysb.edu/ verbaarschot/lecture/

[W] H. Widom, On the relation between orthogonal, symplectic and unitary matrix ensembles. J. Statist. Phys. 94 (1999), 347-363.

Deift: Department of Mathematics, Courant Institute of Mathematical Sciences, New York University, New York, NY 10012

E-mail address: deift@cims.nyu.edu

Gioev: Department of Mathematics, Courant Institute of Mathematical Sciences, New York University, New York, NY 10012, and Department of Mathematics, UniverSITY OF Rochester, Rochester, NY 14627

E-mail address: gioev@cims.nyu.edu 